\title{
Du verbe au nom et du nom au verbe Syntaxe et sémantique des mașdars en arabe standard
}

\author{
FAYSSAL TAYALATI (Université Lille3, France)
}

\begin{abstract}
The article describes the properties of deverbal nouns (mașdars) in Standard Arabic. Prior accounts identify the following type (qasfu l-'aduwwi li-l-madinati), among others, but neglect the mașdar that introduces its internal argument as a direct complement in the genitive case and its external argument as a prepositional adjunct (tahrīiru l-madinati 'alä yadi l-ğayši ). We argue that these two types reflect two different conceptualizations of 'events': bound-events, which describe a change that has taken place in the nature of a substance represented by the internal argument; and unbound-events, which describe a change in the relationship between the internal and external arguments.

Within the lexical decomposition model, we propose a semantic basis for explaining constraints on direct transitive verbs according to (i) the type of mașdars they form; (ii) the possibility of deriving a resultative passive participle (ism al-maf' $\bar{u}$ ) ; and (iii) the alternation, for some verbs, between a causative and non-causative use without any morphological variation.
\end{abstract}

Keywords: lexical decomposition, deverbal noun (mașdars), (un)bound-event.

\section{Introduction}

Nombreux sont les travaux ayant pour objet l'étude des propriétés syntaxiques et/ou morphologiques des mașdars en Arabe Standard (AS), mais rares sont ceux qui traitent de manière approfondie du sens des mașdars et notamment du lien entre la structure lexicoconceptuelle du verbe et le mașdar qui lui est morphologiquement apparenté. Le présent travail se propose de revenir, dans un premier temps sur les propriétés linguistiques des mașdars et de compléter, selon deux aspects précis, les descriptions proposées dans des travaux existants. Il sera question de compléter à la fois la typologie des mașdars de même que leurs descriptions linguistiques. En effet, on peut remarquer que certains types de maṣdars ne sont guère évoqués ou étudiés de manière approfondie, comme c'est le cas dans le travail de FASSI FEHRI (1993) (repris et discutés depuis par KREMERS (2003, 2007) et BARDEAS (2009)) qui insiste(ent), en citant d'autres exemples, sur les propriétés des types illustrés sous (1), en négligeant celui illustré en (2), i.e. celui dans lequel le mașdar prend comme premier complément dans un construct state ${ }^{1}$ le syntagme qui correspond à

1 Au lieu de faire référence à cette construction par la dénomination française 'état construit', nous optons pour la terminologie anglo-saxonne pour éviter toute confusion avec la catégorie sémantique des états, et notamment les états résultants pour lequel le terme 'état' est mobilisé. 
l'argument interne du verbe et non pas celui qui correspond à son argument externe comme en $(1 b, c)$ :

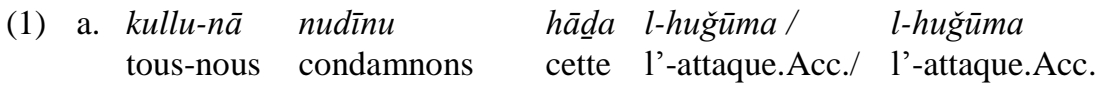

« Nous condamnons tous cette attaque / l'attaque»

b. qasfu l-'aduwwi li-l-madīnati

bombardement.Nom. l'-ennemi.Gén. de-la-ville.Gén.

laylan hallafa damāran kabīran

nuit a causé dégât.Acc. grand.Acc.

«Le bombardement de l'ennemi sur la ville de nuit a causé de grands dégâts »

c. qaśfu l-'aduwwi l-madinata laylan

bombardement.Nom. l'-ennemi.Gén. la-ville.Acc. nuit

hallafa damāran kabīran

a cause dégât.Acc. grand.Acc.

«Le bombardement de l'ennemi sur la ville de nuit a causé de grands dégâts ${ }^{2}$ »

(2)
qasfu
l-madinati (min tarafi l-'aduwwi)
bombardement.Nom. la-ville.Gén. (de part 1-'ennemi)
hallafa iddata daḥ̂̄yā
a causé plusieurs victimes
«Le bombardement de la ville (par l'ennemi) a causé plusieurs victimes »

Les seuls travaux qui incluent dans l'étude le type illustré par (2) en le confrontant à celui sous (1b) sont DAHECH $(2007,2008)$ mais présentent l'inconvénient (i) de négliger celui sous (1c) à peine mentionné et décrit comme exprimant un événement et surtout (ii) n'identifient pour celui en (2) qu'un seul sens alors qu'il peut en avoir deux (signifier une action ou un événement, deux concepts liés mais totalement différents) et (iii) reversent à tort le déverbal sous (1b) dans la catégorie des noms d'événement complexe (selon la terminologie de GRIMSHAW (1990)) le dotant par conséquent d'une structure argumentale alors qu'un ensemble de faits linguistiques montre qu'il n'en n'a pas.

La première partie de cet article aura pour objectif de revenir sur la typologie des mașdars pour la compléter en y incluant le type illustré sous (2) de même que les mașdars en lien avec une base verbale intransitive :

$$
\begin{array}{lll}
\text { rāqșu } & \text { l-fatāti } & \text { muțīrun } \\
\text { danse.Nom. } & \text { la-fille.Gén. } & \text { attirant.Nom. } \\
& \text { «La danse de la fille est excitante » }
\end{array}
$$

2 L'équivalent exact de cet exemple serait le gérondif anglais qui mobilise un déverbal dans le même contexte (The ennemy's bombing the city) là où le français par exemple aurait recours à un participe présent. 
(4)

$$
\begin{array}{llll}
\text { tahyu }^{3} & \text { l-laḥmi } & \text { staǵraqa } & \text { nișfa sātin } \\
\text { cuisson.Nom. } & \text { la-viande.Gén. } & \text { a duré } & \text { demie heure }
\end{array}
$$

«La cuisson de la viande a duré une demie heure »

La deuxième partie de l'article revient sur la syntaxe des mașdars, notamment ceux formés à partir des verbes intransitifs. L'intégration de ces derniers dans l'étude permet de déterminer le type de projection à partir de laquelle sont formés les maṣdars sous (2). La troisième partie de l'article est réservée aux verbes d'action transitifs directs tels que ceux en (5) et (6) avec comme question principale celle de savoir s'il est possible d'avancer une explication sémantique, en partant de la décomposition lexico-conceptuelle des verbes concernés, qui permettrait de mettre en rapport le sens des verbes avec (i) le type de mașdar formé et (ii) la possibilité pour les verbes concernés d'entrer ou non dans une alternance causative/non causative sans changement morphologique. Si l'on prend comme exemple les deux verbes suivants :

$$
\begin{aligned}
& \text { al-ğayšu yaqșifu l-masğida } \\
& \text { l'-armée.Nom. bombarde la mosquée.Acc. } \\
& \text { «L'armée bombarde la mosquée » }
\end{aligned}
$$

$$
\begin{array}{lll}
\text { t-țabbāhhu } & \text { yațhū } & \text { l-laḥma } \\
\text { le-cuisinier.Nom. } & \text { cuit } & \text { la-viande.Acc. } \\
\text { «Le cuisinier cuit la viande » }
\end{array}
$$

même s'il est question dans les deux phrases de verbes d'action a complément direct, seul le second entre dans une construction non causative sans variation morphologique comme le montrent les exemples contrastés ci dessous :

$$
\begin{array}{ll}
\text { *l-masğidu } & \text { yaqșifu } \\
\text { la mosquée.Nom. } & \text { bombarde } \\
\text { l-laḥmu } & \text { yațhū } \\
\text { la-viande.Nom. } & \text { cuit } \\
\text { «La viande cuit » } &
\end{array}
$$

Nous soutiendrons l'idée que seuls les verbes qui possèdent une structure lexicoconceptuelle complexe illustrée sous (9a), et non pas simple (9b), dans laquelle (i) est enchâssée une proposition signifiant le changement d'état de l'entité affectée par l'action de l'agent et (ii) dont une phase de l'action se déroule sans l'intervention de l'agent, autorisent ce type d'alternance :

\section{(9) a. AGIR SUR (x,y) DE SORTE QUE (DEVENIR (ETAT (y) $)^{4}$}

3 Il s'agit d'un mașdar en rapport avec l'emploi intransitif du verbe et non avec son emploi transitif.

4 Nous suivons l'analyse proposé par VAN DE VELDE (manuscrit 2012) pour les verbes français et adoptons les structures lexico-conceptuelles que l'auteur propose. Elles permettent d'avancer une 
b. AGIR MANIERE SUR (x,y)

Les deux structures proposées, outre qu'elles expliquent l'impossibilité pour certains verbes tels que kasara "casser » d'entrer dans une alternance causative/non causative car possédant la condition (i) mais pas la condition (ii), permet également d'expliquer les contraintes sur pèsent sur les nominalisations de ce type de verbes, de même que sur la formation de participes passés à sens résultatif (asmāa $l$-maf $\bar{u} \bar{u} l)$.

\section{Les mașdars: une classe non homogene}

Il est bien connu que les mașdars, qu'ils dénotent un objet concret ou abstrait, sont des noms (en rapport avec une base verbale). Comme il a été remarqué dans la littérature (FASSI FEHRI (1993) ; KREMERS (2003, 2007) ; BARDEAS (2009) entre autres), à l'instar des noms communs, ils se déclinent en cas, au nominatif quand ils sont sujets (10a), à l'accusatif lorsqu'ils dépendent d'un verbe transitif direct (10b) et au génitif lorsqu'ils sont dépendants d'une préposition (10c) :

\begin{tabular}{|c|c|c|c|}
\hline (10) a. & $\begin{array}{l}\text { qasfu } \\
\text { bombardement.Nom. }\end{array}$ & $\begin{array}{l}\text { l-madinnati } \\
\text { la-ville.Gén. }\end{array}$ & $\begin{array}{l}\text { tamma } \\
\text { s'est produit }\end{array}$ \\
\hline & $\begin{array}{ll}\text { l-'āširati } & \text { laylan } \\
\text { dix heures } & \text { nuit }\end{array}$ & & \\
\hline
\end{tabular}

«Le bombardement de la ville s'est produit aux environs de $22 \mathrm{~h} »$

b. nurīdu qașfa l-madīnati

nous voulons bombardement.Acc. la-ville.Gén.

« Nous voulons bombarder la ville»
c. qāma l-amrīkiyyūna bi-qasfi l-madinati ont procédé les américains Prép.-bombardement.Gén. la-ville.Gén.
«Les américains ont procédé au bombardement de la ville »

Ces exemples mettent en avant une deuxième propriété nominale des mașdars. Accompagnés d'un complément, ce dernier se voit assigner le cas génitif (même s'il correspond à l'argument interne du verbe de base) au même titre que s'il était dépendant d'un nom (concret) ordinaire (11). Le cas génitif attribué au complément est l'indice d'une complémentation clairement nominale :

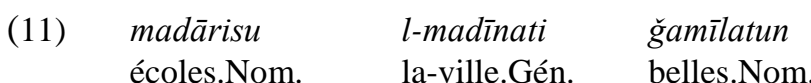

«Les écoles de la ville sont belles »

explication générale à certains phénomènes traités comme isolés les uns des autres. Sur l'adéquation du Primitif AGIR SUR et non de celui de CAUSE proposé par LEVIN et RAPPAPORT HOVAV (1995, 2005), voir VAN DE VELDE (2011). 
Enfin $^{5}$, et à condition que les référents ne soient pas massifs, les mașdars admettent la pluralisation (y compris le duel) et par voie de conséquence une détermination, aussi bien par un article défini qu'indéfini :

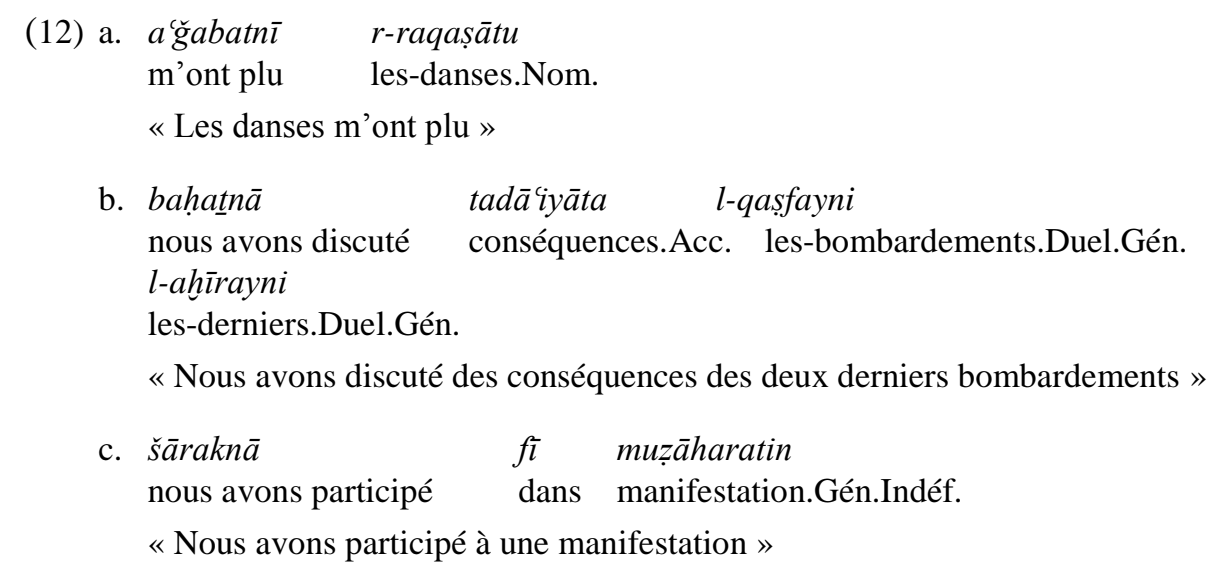

Cependant, depuis le travail pionnier de FASSI FEHRI (1993) dans lignée de GRIMSHAW (1990), on distingue les déverbaux à propriétés verbales et les déverbaux à propriétés nominales (à sens événementiel ou résultatif). Les premiers, contrairement aux seconds, retiennent la structure aspectuelle du verbe correspondant et possèdent par conséquent une structure argumentale. Une synthèse des principales propriétés relevées par Grimshaw pour les deux types de déverbaux est donnée ci-dessous :

(13) Complex event nominals

(i) Event reading, (ii) Obligatory arguments, (iii) Agent-oriented modifiers, (iv) Implicit argument control, (v) Compatible with aspectual modifiers like in three hours, (vi) Modifiers like frequent, constant only with singular, (vii) by phrases are arguments, (viii) Must be singular, (ix) Must be definite

(14) Simple event and Result nominals

(i) No event reading, (ii) No obligatory arguments, (iii) No agent oriented modifiers, (iv) No implicit argument control, (v) Not compatible with aspectual modifiers, (vi) Modifiers like frequent, constant with plural, (vii) by phrases are not arguments, (viii) May be plural, (ix) May be indefinite.

L'application de ces tests à l'AS aboutit à une typologie ternaire qu'on retrouve dans les travaux de FASSI FEHRI (1993), KREMERS (2003) ; BARDEAS (2009) entre autres. Cette typologie reflète le degré de nominalisation des maṣdars en question, du plus nominal au moins nominal. Ils sont présentés rapidement sous une autre terminologie qui oppose les noms issus d'une nominalisation achevée et les noms issus d'une nominalisation

5 Pour d'autres arguments, voir FASSI FEHRI (1993), KREMERS (2003) et BARDEAS (2009). 
inachevée. La terminologie est empruntée à VAN DE VELDE (2006a) qui a le mérite d'évacuer de l'appellation le concept «événement» qui sera développé et défini plus loin (partie 4). Une fois défini, ce concept sera mobilisé pour distinguer, au sein des mașdars qui dénotent un événement, ceux qui expriment un événement lié ayant pour vocation de décrire le changement survenu dans le référent de leur unique argument (l'argument interne du verbe correspondant) de ceux qui expriment un événement libre délié de toute substance.

\subsection{Synthèse du travail de FASSI FEHRI 1993 ; KREMERS 2003, 2007 ; Suzanne BARDEAS 2009}

Les auteurs distinguent trois types de mașdars. Les plus nominaux de par leurs propriétés morpho-syntaxiques sont ceux illustrés sous (15). En effet, ils se présentent dépouillés de structure argumentale et acceptent plusieurs types de déterminants :
(15) kullu-nā nudīnu
hāda l-huğūma
/l-huğūma
tous-nous condamnons
cette l'-attaque.Acc.
/l'-attaque.Acc.

« Nous condamnons tous cette attaque / l'attaque»

Ils acceptent la pluralisation (y compris le duel) à condition bien évidemment qu'ils soient non homogènes, de même qu'une modification (pour exprimer la manière) adjectivale (17) comme pour les noms ordinaires (voir aussi Kremers et Bardeas) :

(16)
'itirāfātu-hu
confession.Fém.Plu.Nom.-Pro.Poss.
$\dot{g} a y r u$
pas
muqni'atin
convaincantes.Gén.

(17) hāda l-itirāfu

ce le-confession.Nom. étrange.Nom.

«Cette confession est étrange » (cf. Fassi Fehri 1993 : 237)

Deux autres déverbaux distingués par les auteurs auxquels est attribué un sens événementiel sont illustrés par :
'aqlaqan̄̄ ntiqā $d u$
r-ră̆uli
l-mašrū'a
m’a énervé critique.Nom. l'-homme.Gén. le-projet.Acc.
«The man's criticizing the project annoyed me »
'aqlaqa-n̄̄ ntiqāadu r-rağuli
li- l-mašrūi
a énervé-moi critique l'-homme.Gén. de le-projet.Gén.
« La critique par l'homme du projet m'a énervé »

Celui illustré en (18), équivalent au gérondif anglais accompagné d'un complément direct, est encore verbal puisqu'il assigne, à l'instar du verbe correspondant intaqada « critiquer », le cas accusatif au deuxième argument du verbe correspondant. A cela s'ajoute la 
possibilité d'une modification de manière de type adverbial (prép.+nom) comme c'est le cas avec le verbe correspondant (cf. Fassi Fehri 1993: 241) :

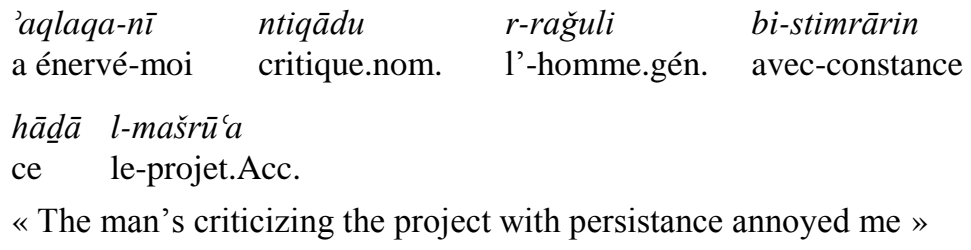

Concernant le sens, et contrairement à ce que soutiennent les auteurs mentionnés ci-dessus, le mașdar équivalent au gérondif anglais accompagné d'un complément direct à l'accusatif, lequel a une lecture active puisqu'il décrit ce que fait l'agent, réfère à une action et non à un événement. Le propre de l'action est d'être prédiquée d'un agent alors qu'un événement quel qu'il soit, et nous reviendront plus loin sur les deux types que la langue permet d'exprimer, a comme particularité d'être coupé d'un agent, mettant ainsi l'accent, soit sur un changement quelconque mais présenté du point de vue du patient dans le cas des événements non liés, soit sur le changement de l'état du thème dans le cas des événements liés. Dans le cas des prédicats exprimant une action, l'agent a le statut syntaxique d'argument et non d'adjoint. Pour revenir au mașdar illustré sous (18), il est intéressant de remarquer que le syntagme r-rağuli (l'homme) entretient un rapport argumental avec le mașdar puisque l'argument interne au cas accusatif ne peut être licencié qu'en la présence de l'argument externe 'agent' :

$$
\begin{array}{lll}
\text { *'aqlaqan̄i } & \text { ntiqāa } & \text { l-mašrū̄a } a \\
\text { m'a énervé } & \text { critique.Nom. } & \text { le-projet.Acc. }
\end{array}
$$

Le deuxième argument à l'appui du sens actionnel et non événementiel de ce type de mașdar est tiré de l'impossibilité d'être employé avec des prédicats verbaux posant l'existence des événements :

$$
\begin{aligned}
& \text { (22) a. *intiqādu r-rağuli l-mašrū'a tamma lbārihata } \\
& \text { critique.Nom. l'-homme.Gén. le-projet.Acc. a eu lieu hier } \\
& \text { b. *qasfu 'aduwwi l-madinata waqa'a } \\
& \text { bombardement.Nom. l'-ennemi.Gén. la-ville.Acc. a eu lieu } \\
& \text { lbārihata } \\
& \text { hier }
\end{aligned}
$$

L'exemple (22b) contraste fortement avec les suivants qui mobilisent le même nom tête à la différence que l'agent cette fois-ci est supprimé. La suppression de ce dernier favorise l'émergence d'un autre sens, celui d'un événement basé sur un changement identifié soit par l'argument interne (23a) soit délié de ce dernier (23b). La suppression de l'agent rend les syntagmes aptes à se combiner avec les verbes posant l'existence d'un événement : 
(23) a. qașfu l-madīnati waqa'a lbārihata $^{6}$

bombardement.Nom la-ville.Gén. a eu lieu hier

«Le bombardement de la ville a eu lieu hier »

b. al-qașfu waqa'a lbārihata

le-bombardement.Nom. a eu lieu hier

«Le bombardement a eu lieu hier»

\subsection{Les déverbaux à sens événementiel : deux types de nominalisation, deux types d'événement}

Le deuxième mașdar à propriétés clairement verbales, non étudié de manière exhaustive par les auteurs mentionnés ci-dessus, est celui illustré sous (24) dont les propriétés remarquables, aussi bien syntaxiques que sémantiques, émergent en le contrastant avec celui sous (25) à propriétés clairement ${ }^{7}$ nominales cette fois-ci :
(24) a. qasfu
l-madīnati min țarafi
l-'aduwwi
bombardement.Nom. la-ville.Gén. de part l'-ennemi.Gén.

«Le bombardement de la ville par l'ennemi »

b. tahrīru

l-madīnati 'alā yadi l-ğayši

l-ḥurri

libération.Nom. la-ville.Gén. de main l'-armée.Gén. le-libre.Gén.

«La libération de la ville de la part de l'armée libre »

c. ta'dīlu d-dustūri min tarafi

modification.Nom. la-constitution.Gén. de part

l-hukūmati

le-gouvernement.Gén.

«La modification de la constitution de la part du gouvernement »

(25) a. qașfu l-'aduwwi li-l-madinati

bombardement.Nom l'-ennemi.Gén. Prép.-la-ville.Gén.

«Le bombardement ennemi sur la ville »
b. tahrīiru
l-’̆gyši
l-hurri
li-l-madinati
libération.Nom. l'-armée.Gén. le-libre.Gén. Prép.-la- ville.Gén.

«La libération de l'armée libre de la ville »

6 Le masdar qasf accompagné de l'argument interne du verbe correspondant exprime également le concept d'action (cf. 1.2.1.). Seul le contexte et le choix des prédicats permettent la désambiguïsation.

7 Mis à part DAHECH (2008) qui le reverse dans la catégorie des noms d'événements complexes, i.e. ayant retenu les propriétés aspectuelles et argumentales du verbe correspondant, tous les auteurs, même s'ils ne relèvent pas toutes les propriétés de ce type, insistent à juste titre sur son statut clairement nominal, et par conséquent le traitent comme un nom d'événement simple selon la terminologie de Grimshaw (1990). 


\section{c. ta'dīlu l-hukūmati $^{8} \quad$ li-d-dustūri \\ modification.Nom. le-gouvernement.Gén. Prép.la-constitution.Gén.}

«La modification du gouvernement de la constitution »

La comparaison des deux types de nominalisation a été menée pour le français par VAN DE VELDE (2006a) qui a mis en avant un faisceau de propriétés qui convergent vers la conclusion que les deux nominaux réfèrent à deux types d'événement ontologiquement différent. Un travail relativement récent (DAHECH 2008) applique une partie des tests relevés par VAN DE VELDE aux deux types de déverbaux, mais reste critiquable sur trois points (au moins) :

- l'auteur n'identifie qu'un seul sens pour les maṣdars en (24) alors qu'ils en possèdent deux ;

- reverse ceux sous (25) dans la catégorie des noms d'événement complexe. Cependant, plusieurs propriétés montrent que les déverbaux en (25), contrairement à ceux en (24), sont issus d'une nominalisation achevée et ne possèdent plus de structure argumentale ;

- et ne propose pas de base sémantique qui pourrait contribuer à systématiser la formation des masdars.

\subsubsection{Les masdars à lecture passive, issu d'une nominalisation inachevée (sens d'action, servant de base aussi à une lecture événementielle).}

Le mașdars sous (24) possèdent des propriétés clairement verbales et peuvent exprimer à la fois une action ou un événement. Seul le contexte désambigüise l'interprétation. Ils sont identifiables par l'ordre des arguments : le mașdar prend comme premier argument au génitif celui qui correspond à l'argument interne du verbe. L'argument 'agent' est relégué à la deuxième position et est introduit par les locutions prépositionnelles min tarafi/'alā yadi (de la part de) qui introduisent en AS les complément d'agent dans une construction verbale passive ${ }^{9}$ :

$\begin{array}{lll}\text { (26) a. qușifa } & \text { l-masğidu } & \text { (min țarafi /'alä yadi } \\ & \text { a été bombardé la-mosquée.Nom. } & \text { (de part }\end{array}$

8 Les exemples sous $(25 \mathrm{~b}, \mathrm{c})$ montrent une différence majeure entre l'AS et le français quant aux possibilités de nominalisation. En français (cf. VAN DE VELDE 2012, manuscrit), le verbe modifier n'autorise qu'une nominalisation inachevée du type la modification de la constitution par le gouvernement et non une nominalisation achevée *la modification du gouvernement de/sur la constitution. Les verbes transitifs directs en AS, du moins ceux en lien avec les mașdars donnés comme exemples, autorisent les deux types de nominalisation. La différence entre le couple tahrir-harrara « libération-libérer » et qasf-qasafa «bombardement-bombarder » est à chercher du côté des participes passés comme nous le préciserons plus loin. L'intégration dans l'étude des participes passés de même que la possibilité ou non d'entrer dans une alternance causative/non causative sans changement morphologique affinera la description des prédicats en question.

9 Le rapprochement que nous établissons entre les mașdars sous (24) et les verbes correspondants dans la version passive (26) n'implique pas que les premiers sont issus d'un processus de passivation. Comme nous le verrons plus loin, ce type de mașdar -avec les propriétés et (un des effets de sens) relevés dans la partie (2.2.1.)- croise ceux issus d'une classe de verbes intransitifs qui n'admet pas la passivation. Reste à déterminer ce qui rapproche syntaxiquement et sémantiquement les deux types de mașdars. 
murtaziqati bašār)

mercenaires.Gén. Bachar

«La mosquée a été bombardée (par les mercenaires de Bachar) »

b. hurrira l-baladu (min țarafi/'alā yadi l-ğayši

a été libéré le-paysNom. (de part de l'-armée.Gén.

l-hurri)

le-libre.Gén.)

«Le pays a été libéré (par l'armée libre) »

La deuxième propriété qui appuie le rapprochement entre les mașdars sous (24) et les verbes correspondants dans leur version passive et le statut argumental de l'argument interne. Dans une passivation (26), alors que le complément d'agent est facultatif, l'argument interne du verbe, au nominatif, est obligatoire. Sa mention est obligatoire pour licencier la présence du complément d'agent qui a le statut d'un adjoint spécifiant l'instigateur de l'action :

$$
\begin{aligned}
& \text { (27) a. *qușifa min țarafi/'alāyadi murtaziqati Bašār } \\
& \text { a été bombardé de part mercenaires.Gén. Bašār } \\
& \begin{array}{llll}
\text { b. *hurrira min } & \text { tarafi /'alā yadi } & \text { l-ğayši } & \text { l-ḥurri }{ }^{10} \\
\text { a été libéré de } & \text { part } & \text { l'-armée.Gén. } & \text { le-libre.Gén. }
\end{array}
\end{aligned}
$$

Les maṣdars issus d'une nominalisation inachevée présentent la même propriété. Le complément d'agent est facultatif (28) tandis que l'argument interne est obligatoire. La présence du complément d'agent est en effet conditionnée par la présence de l'argument interne comme le montre l'agrammaticalité des énoncés sous (29) :
(28) a. qașfu l-madīnati
(min tarafi l-'aduwwi)
bombardement.Nom. la-ville.Gén. (de part l'ennemi)
hallafa iddata dahāay $\bar{a}$
a cause plusieurs victimes
«Le bombardement de la ville (de la part de l'ennemi) a causé plusieurs victi- mes»
b. ta'dīlu d-dustūri (min tarafi
modification.Nom. la-constitution.Gén. de part

$\begin{array}{lll}\text { l-h̆ukūmati) } & \text { askata } & \check{s} \text {-šārica } \\ \text { le-gouvernement.Gén. } & \text { a calmé } & \text { la-rue.Acc. }\end{array}$
«La modification de la constitution (de la part du gouvernement) a calmé la rue »

10 Ces exemples seraient acceptables dans un dialogue si l'argument interne avait été mentionné dans le contexte précédent. 
(29) a. *qașfu min tarafi l-'aduwwi hallafa

bombardement.Nom. de part l'ennemi a causé

iddata dahāāā

Plusieurs victimes

b. *ta'dìlu min tarafi l-ḥukümati

modification.Nom. de part le-gouvernement.Gén.

askata š-šāía

a calmé la-rue.Acc.

Comme dans le cas d'un prédicat verbal (30), ou d'un mașdar équivalent au gérondif anglais (à propriétés verbales, cf.1.1.), les modifieurs indiquant l'aspect ou la manière de l'action faite par l'agent sont de forme adverbiale et non adjectivale, ce qui s'explique par la nature verbale du mașdar issu d'une nominalisation inachevée (31) :

(30)

$\begin{array}{llll}\text { qușifa } & \text { l-maš̆ğidu } & \text { bi-šarāsatin/ } \\ \text { a été bombardée } & \text { la-mosquée.Nom. } & \text { avec-sauvagerie/ } \\ \text { *š-šarisu min } & \text { tarafi murtaziqati } & \text { bašăar } \\ \text { le-sauvage de } & \text { part mercenaires.Gén. } & \text { Bachar }\end{array}$

«La mosquée a été sauvagement bombardée par les mercenaires de Bachar»

b. 'udīla di-dustūru bi-sur'atin/*s-sarīú

a été modifiée la-constitution.Nom. avec-rapidité/*le-rapide

min tarafi l-ḩukūmati li-iskāti š-šārici

de part le-gouvernement.Gén. pour-calmer la-rue.Gén.

«La constitution a été modifiée rapidement par le gouvernement pour calmer la rue »

(31) a. qașfu l-madīnati bi-šarāsatin/ *š-šarisu

bombardement.Nom. la-ville.Gén. avec-sauvagerie/ *le-sauvage

min țarafi l-'aduwwi ḩallafa iddata dahāya

de part l'-ennemi.Gén. a causé plusieurs.Acc. victimes.Acc.

«Le bombardement sauvage de la ville de la part de l'ennemi a causé plusieurs victimes »

b. ta'dīlu d-dustüri bi-sur'atin/

modification.Nom. la-constitution.Gén. avec-rapidité/

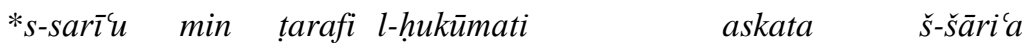

*le-rapide de part le-gouvernement.Gén. a calmé la-rue.Acc.

«La modification rapide de la constitution de la part du gouvernement a calmé la rue » 
Concernant le sens de ce type de mașdar, il faut préciser qu'il peut servir de base à la formation d'une phrase où ce qui est exprimé est de l'ordre, soit de l'action, soit de l'événement. La désambigüisation se fait en contexte. L'exemple sous (23) reproduit cidessous, de même que (32b) expriment un événement comme le montre la combinaison possible avec, soit un verbe posant l'existence d'un événement (32a) soit un nom classifieur qui rattache le sujet à la classe des événements (32b) :

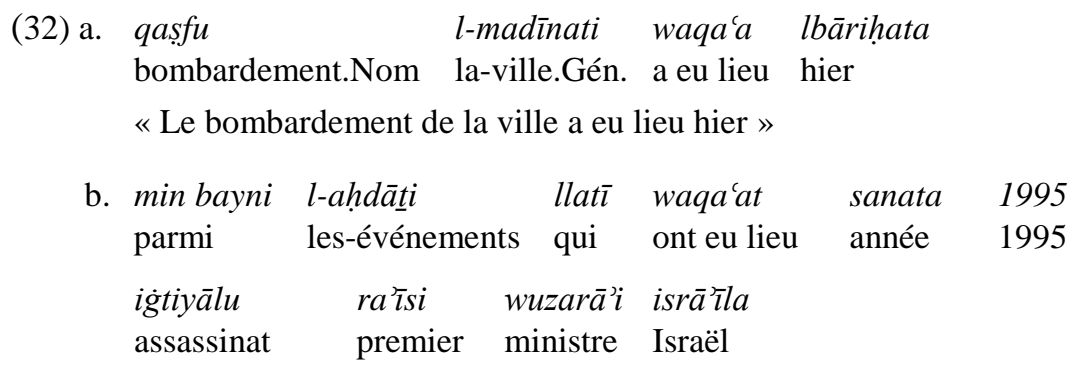

«Parmi les événements qui eu lieu en 1995, l'assassinat du premier ministre israélien »

Cependant, les deux mașdars peuvent également servir de base à l'expression d'une action. L'action se distingue de l'événement par le fait que le premier concept est toujours prédiqué d'un agent. Sous (33), les deux syntagmes (accompagnés de leur unique argument, celui qui correspond à l'argument interne du verbe correspondant) sont combinés avec des prédicats qui les présentent cette fois-ci, comme des actions, puisqu'ils sont rattachés à un agent, contrairement a la situation illustrée sous (32) :
(33) a. $q \bar{a} m a^{1}$
l-'aduwwu
bi-qasfi
l-madinati
a procédé l'ennemi.Nom. Prép.-bombardement.Gén. la-ville.Gén.
«L'ennemi a procédé au bombardement de la ville »
b. hāwala l-mutašaddidu iğtiyala rā̌si wuzarāì isrāî̀la a tenté l'-extrémiste.Nom. assassinat.Acc. premier ministre Israël
«L'extrémiste a tenté d'assassiner le premier ministre israélien »

Les deux sens identifiés pour ce type de mașdar (à propriétés verbales), outre qu'ils expliquent le choix des prédicats exemplifiés sous (32) et (33), soulèvent une question importante qui concerne particulièrement le type d'événement exprimé par les mașdars issus d'une nominalisation inachevée (à propriétés clairement nominales), puisque les masdars qui seront présentés ci-dessous expriment (exclusivement) à leur tour des événements. La troisième partie de l'article, basée sur la description lexico-conceptuelle des verbes, tentera d'apporter une réponse à la question de l'existence de deux modes pour l'expression d'événements par le biais des masdars.

11 Cf. Van de Velde (2006a) pour le choix des verbes supports en français. 


\subsubsection{Le déverbal issu d'une nominalisation achevée (à sens événementiel).}

Le type de maṣdar à sens uniquement événementiel, est illustré sous :

(34) a. qașfu l-'aduwwi li-l-madinati

bombardement.Nom. l'-ennemi.Gén. de-la-ville.Gén.

laylan hallafa damāran kabīran

nuit a causé dégât.Acc. grand.Acc.

«Le bombardement nocturne de l'ennemi sur la ville a causé de grands dégâts »

b. 'iqtirābu ziyārati l-maliki li-l-madīnati

approche.Nom. visite.Gén. le-roi.Gén. de-la-ville.gén.

«L'approche de la visite du Roi de la ville »

L'ordre des arguments et leur mode d'introduction sont révélateurs des propriétés nominales ${ }^{12}$ des mașdars. Dans les deux cas, le nom prend comme premier complément au génitif celui qui correspond à l'agent (argument externe), et l'argument interne est relégué en deuxième position, introduit indirectement par une préposition qui lui attribue le cas génitif.

Alors que l'argument interne du verbe correspondant est obligatoire quand il est dépendant d'un mașdar à propriétés verbales (cf. partie 2.2.1.), aussi bien en (35a) qu'en (35b), il est facultatif et peut être omis sans affecter la grammaticalité des phrases, ni changer le sens du déverbal :
(35) a. qasfu l-'aduwwi
hallafa
damāran
bombardement.Nom. l'ennemi.Gén. a causé dégat.Acc.
kabìran
grand.Acc.
« Le bombardement de l'ennemi a causé de grands dégâts »
b. 'iqtirābu ziyārati l-maliki approche.Nom. visite.Gén. le-roi.Gén.
«L'approche de la visite du Roi »

Des propriétés supplémentaires révélatrices de la nature nominale des mașdars en question se manifestent lorsque le complément 'agent' est introduit dans la phrase sous forme adjectivale. En effet, tant que l'agent est de forme nominale, on pourrait croire qu'aussi bien $l$-'aduwwi «l'ennemi » que l-maliki «le Roi» sont des arguments. Cependant, contrairement aux mașdars décrits en (1.2.1.) qui possèdent une structure argumentale, ceux-ci n'en possèdent pas puisque l'argument externe du verbe correspondant peut être

12 Voir également les travaux de FASSI FeHri (1993) ; KrEMERS (2003) et BARDEAS (2009). Nous insistons plutôt sur les propriétés non révélées par les auteurs mais qui convergent vers les mêmes conclusions, i.e. les propriétés nominales de ce type de déverbal. 
introduit dans les mêmes phrases (en la présence ou non du syntagme l-madinati «la ville ») sous la forme d'un adjectif de groupe :

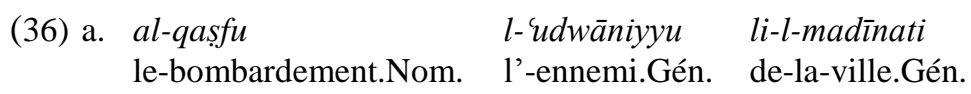

$\begin{array}{llll}\text { laylan hallafa damāran } & \text { kabīran } \\ \text { nuit } & \text { a cause } & \text { dégat.Acc. } & \text { grand.Acc. }\end{array}$

«Le bombardement ennemi de nuit sur la ville a causé de grands dégâts »

b. 'iqtirābu z-ziyārati malakiyyati li-l-madīnati approche.Nom. la-visite.Gén. la-royale.Gén. de-la-ville.gén.

«L'approche de la visite royale de la ville »

Son introduction sous la forme d'un adjectif (de groupe) est la preuve de la nature nominale du mașdar, et du statut non argumental du complément 'agent'. Ces deux propriétés sont respectivement appuyées par le fait que, d'une part les modifieurs aspectuels et de manière sont également introduits sous une forme adjectivale et non adverbiale $(37)^{13}$, et d'autre part l'incapacité du référent de l'agent, lorsqu'il est de forme adjectivale, à lier une anaphore comme le montrent les contrastes sous $(38)^{14}$ :

(37)
qasfu
l-'aduwwi
$\check{s}$-šarisu/
*bi-šarāsatin
bombardement.Nom. l'-ennemi.Gén. le-sauvage/ *avec-sauvagerie

li-l-madīnati laylan hallafa damāran kabìran

de-la-ville.Gén. nuit a causé dégât.Acc. grand.Acc.

«Le bombardement sauvage de l'ennemi sur la ville de nuit a provoqué de grands dégâts »

b. al-qasfu l-'udwāniyyu l-mustamirrul *bi-stimrārin

le-bombardement.Nom. l'-ennemi.Gén. le-continu *avec-continuité

li-l-madīnati laylan hallafa damāran kabīran

de-la-ville.Gén. nuit a causé dégat.Acc. grand.Acc.

«Le bombardement ennemi en continu sur la ville a causé de grands dégâts »

(38) a. qașu-hum $\mathrm{i}_{\mathrm{i}}$ li-l-madaniyyīna

attaque.Nom.-Pron.Clit. de-les-civils.Gén.

afqada-hum $_{i} \quad$ mișdāqiyata-hum ${ }_{i}$

a fait perdre.-Pron.Clit. la-crédibilité.Acc.-Pron.Clit.

«Leur bombardement des civils leur a fait perdre leur crédibilité »

13 Cf. FASSI FEHRI (1993) repris dans KREMERS (2005) et BARDEAS (2009).

14 Cf. entre autres Alexiadou et Stavrou (1998); Alexiadou (2001); Markantanatou et OERSNES (2002) ; VAN DE VELDE (2006a, 2012 manuscrit). 


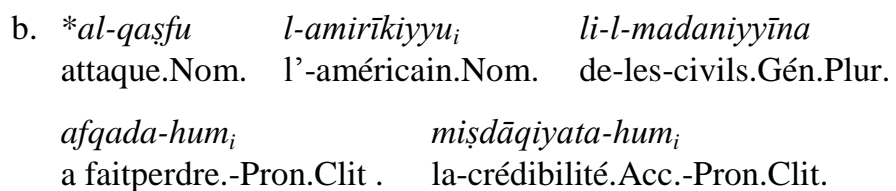

Un fait supplémentaire -corrélé à la réalisation de l'argument externe des verbes correspondant sous la forme d'un adjectif de groupe- est l'apparition d'un déterminant obligatoire pour les mașdars issus de ce type de nominalisation (achevée) :
(39) a. al-qasfu
l-amrīkiyyu
hallafa
le-bombardement.Nom.
l'-amricain.Nom. a causé
damāran kabīran
dégât.Acc. grand.Acc.
«Le bombardement américain a causé de grands dégâts »
b. iqtirābu
z-ziyārati l-malakiyyati
li-l-madīnati
approche.Nom. la-visite.Gén. la-royale.Gén.
de-la-ville.Gén.
«L'approche de la visite royale de la ville»

La détermination appuie la nature nominale des mașdars en question, laquelle est également prouvée par la possibilité d'avoir un pluriel (et un duel). La détermination n'est pas contrainte :

(40)
al-qașafātu
l-amrīkiyyatu
allafat
les-bombardements.Nom.
l'-amricaines.Nom. ont causé
damāran kabīran ${ }^{15}$
dégât.Acc. grand.Acc
«Les bombardements américains ont causé de grands dégâts »
b. katurati
z-ziyāāātu
l-malakiyyatu
devenues nombreuses
les-visites.Nom.
les-royales.Nom.
«Les visites royales sont devenues nombreuses »

Enfin, il est possible de réintroduire dans les exemples ci-dessus l'adjoint l-madīnati «la ville » en l'absence du constituant qui renvoie à l'agent, preuve que les deux n'entretiennent pas de relation argumentale avec le mașdar (41). Aussi bien l'agent que le patient ont le statut d'actant qui spécifient la nature des deux, mais ne constituent pas des arguments, ce qui permet au mașdar issu d'une nominalisation inachevée de s'employer même dépouillé des deux (42) :

\footnotetext{
15 La nature nominale du déverbal en (40a) est également confirmée par la nature du modifieur indiquant la manière : la modification se fait par un adjectif $s$-šarisatu (féroce) et non plus par un adverbe $b i$ šarāsatin (avec férocité).
} 
(41) a. tawāṣalati l-qașafātu 'alā l-madīnati «Les bombardements sur la ville ont continué »

b. katurati z-ziyāratu li-l-madīnati devenues nombreuses les-visites.Nom. de-la-ville.Gén.

«Les visites de la ville sont devenues nombreuses »

(42) a. tawāṣalati l-qașafātu

ont continué les-bombardements.Nom.

«Les bombardements ont continué »

b. katurati z-ziyāratu

devenues nombreuses les-visites.Nom.

«Les visites sont devenues nombreuses »

Etant donné que ce type de mașdar est dépouillé d'arguments, il ne peut dénoter qu'un événement, sachant que l'événement est caractérisé par l'absence d'un agent, lequel s'il est présent, n'est pas pour autant un argument. Que l'agent soit lexicalisé (sous la forme d'un nom (43a) ou d'un adjectif de groupe (43b)) ou qu'il soit absent (43c), les mașdars se combinent avec des prédicats verbaux (ou nominaux classifieurs) qui les présentent comme exprimant des événements :
(43) a. qasfu
l-'aduwwi
(li-l-madinati)
bombardement.Nom.
l'-ennemi.Gén.
(de-la-ville.Gén.)
waqa'a sanata 1960
a eu lieu année 1960
«Le bombardement de l'ennemi (sur la ville) a eu lieu en 1960 »
b. al-qașfu l-'udwāniyyu waqa'a sanata 1960 le-bombardement.Nom. l'-ennemi.Gén. a eu lieu année 1960
«Le bombardement ennemi a eu lieu en 1960 »
c. al-qașfu waqa'a sanata 1960
le-bombardement.Nom. a eu lieu année 1960
«Le bombardement ennemi a eu lieu en $1960 »$

Il est toujours possible de rattacher l'agent au mașdar à la manière d'un argument par le biais d'un prédicat tel que qāma (procéder à). Ce mode de rattachement permet au syntagme constitué de dénoter une action, mais seulement si le thème (i.e. l'argument interne du verbe correspondant) est introduit comme complément argumental au génitif dans un construct state. Comme montré dans la partie (2.2.1), lorsque le mașdar prend comme uniquement argument le thème, il peut exprimer une action et être rattaché à un agent, ce qui est illustré sous (44). Cependant, lorsqu'il s'agit d'un mașdar issu de nominalisation 
achevée, étant donné qu'il ne possède plus d'arguments, il n'existe plus de possibilité de dénoter une action comme le montre l'agrammaticalité de (45):

$$
\begin{array}{llll}
\text { qàma } & \text { l-'aduwwu } & \text { bi-qasfi } & \text { l-madīnati } \\
\text { a procédé } & \text { l'ennemi.Nom. } & \text { Prép.-bombardement.Gén. } & \text { la-ville.Gén. } \\
\text { "L'ennemi a procédé au bombardement de la ville » } &
\end{array}
$$

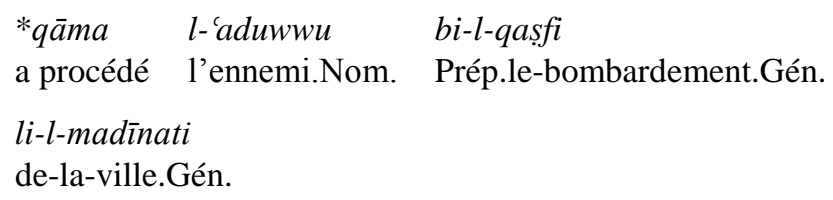

Il ressort de la comparaison que des deux derniers types de mașdars que les premiers ont des propriétés verbales, et les seconds nominales. Les différents mașdars en AS donnent donc un aperçu du spectre des déverbaux incluant trois types fondamentalement différents. La nominalisation inachevée donne lieu à deux types de mașdars a propriétés verbales ; la nominalisation achevée permet la formation d'un mașdar a propriétés nominales, pouvant s'accompagner de compléments-actants, mais pouvant également s'en affranchir et s'employer comme nom ordinaire.

\section{Descriptions syntaxiques des mașdars}

Les analyses syntaxiques que nous rappelons dans un premier temps dans cette partie, aussi différentes soient elles, s'accordent sur le point suivant : le nom, déverbal ou non, forme avec le complément qui le suit, et dans le cas d'un mașdar, qu'il s'agisse d'un argument ou d'un simple actant, un construct state. Les analyses les plus influentes ${ }^{16}$ de cette construction lui assignent une structure parallèle à celle d'une proposition (clause). Les analyses théoriques diffèrent dans les détails mais défendent l'idée que le construct state consiste en un DP (Determiner Phrase) avec une tête vide qui domine la projection lexicale dont la tête, dans le cas des mașdars, est le mașdar lui même (appelé Construct Head). Ce dernier monte et s'incorpore à la tête du DP. Le complément du mașdar (appelé Inner Head) est inséré dans la position Spec. de la projection lexicale (et dans certains travaux dans une projection fonctionnelle intermédiaire), position dans laquelle il reçoit le cas génitif. Le mașdar est son complément s'accordent en définitude selon la relation tête-Specifieur. L'ordre de surface de la construction exemplifiée sous (46a) ne reflète pas l'ordre profond, lequel est représenté sous (46b) :
(46) a.
qasfu l-'aduwwi
bombardement.Nom l'-ennemi.Gén.
«Le bombardement de l'ennemi »

16 Cf. Aoun (1977); Ayyoub (1985); MOHAMMAD (1988); FASSI-FeHRI (1993); OUhAlla (1988, 1994) ; RITTER (1988, 1991, 1993, 1995) ; KREMERS (2003) entre autres. 


$$
\text { b. } \left.\left.{ }_{\mathrm{DP}}[\mathrm{D} \text { D qasfu }][]_{\mathrm{NP}}\left[{ }_{\mathrm{DP}} l \text { 'caduwwi }\right] \mathrm{t}_{\mathrm{i}}\right]\right]
$$

Les deux analyses syntaxiques des mașdars que nous présentons dans cette partie diffèrent aussi bien selon le cadre théorique adopté que selon le processus de formation des mașdars. Elle mettent l'accent sur les deux types illustrés sous :

$$
\begin{array}{lll}
\text { ¿intiqāadu } & \text { r-rağuli } & \text { li-l-mašrūi } i \\
\text { critique.Nom. } & \text { l'-homme.Gén. } & \text { de-le-projet.Gén. }
\end{array}
$$

«La critique par l'homme du projet »

$$
\begin{aligned}
& \text { 'intiqādu } \quad \text { r-rağuli } \quad l \text {-mašrū'a } \\
& \text { critique.Nom. l'-homme.Gén. } \\
& \text { «The man's criticizing the project.Acc. }
\end{aligned}
$$

FASSI FEHRI (1993) défend l'idée qu'un verbe et un affixe nominaliseur à sens événementiel (E.af.) sont projetés dans un construct state verbal et que la combinaison du verbe et de l'affixe change le verbe en nom. La structure sous (49) correspond au type illustré en (47) :

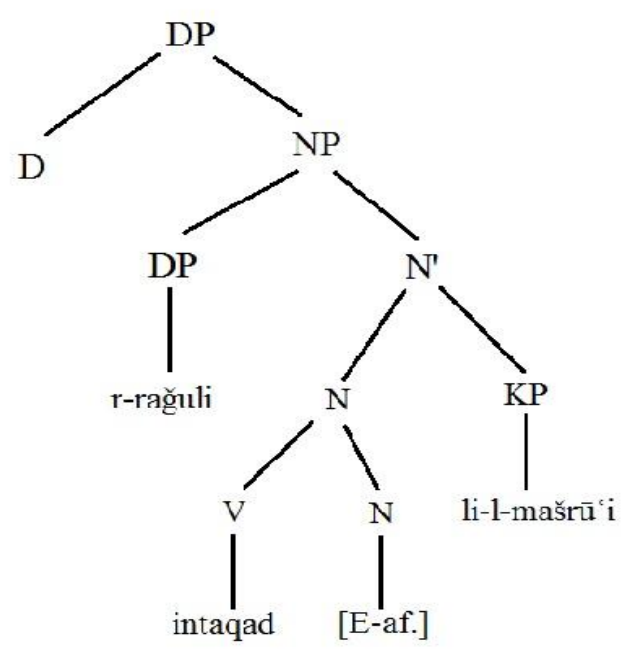

l'affixe s'attache directement au verbe et le convertit en un nom. Ce qui explique les propriétés nominale de ce type de mașdar, à savoir (i) la modification aspectuelle et de manière par des adjectifs et non par des adverbes et surtout (ii) l'incapacité de donner le cas accusatif à l'argument interne du verbe, lequel reçoit le cas génitif par l'intermédiaire d'une préposition étant donné que le syntagme prépositionnel n'est plus dépendant d'un verbe, mais d'un nom.

Le mașdar avec un argument interne à l'accusatif (48) est le résultat de l'attachement de l'affixe nominalisateur à une projection verbale complète contenant, le verbe et ses deux arguments (interne et externe) : 
(50)

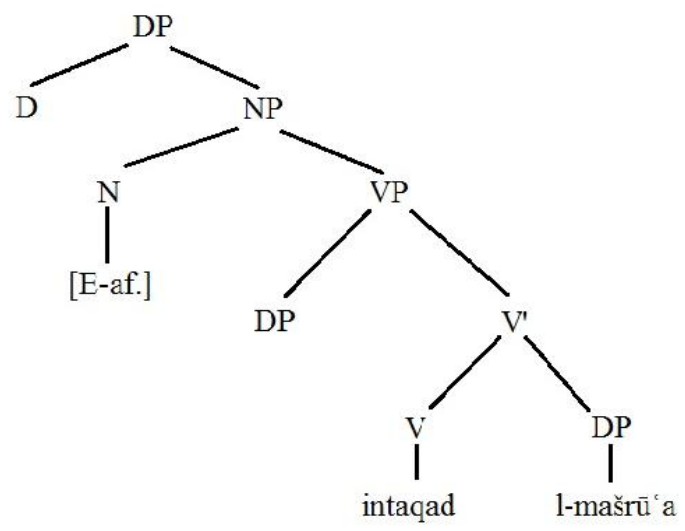

Dans ce deuxième cas, l'affixe nominaliseur s'attache à un VP après que le verbe ait projeté ses arguments. La présence d'une tête verbale explique les propriétés verbales du mașdar formé suite à l'affixation, notamment (i) la modification aspectuelle et de manière par des adverbes et non des adjectifs et (ii) la capacité d'attribuer le cas accusatif à l'argument interne, qui s'explique par la présence d'une tête verbale et d'un argument externe auquel le verbe assigne le rôle d'agent. ${ }^{17}$

KREMERS (2003) propose une analyse dans un cadre minimaliste, et opte pour une dérivation sans affixation. L'auteur soutient l'idée que la dérivation des deux mașdars illustrés cidessus prend place à des niveaux différents. Le mașdar accompagné de l'argument interne à l'accusatif est formé à partir de l'insertion de la racine 'ntqd' dans une projection verbale complète dominée, non pas par la projection $\mathrm{T}$ (responsable de la réalisation de la racine sous la forme d'un verbe) mais par la projection $\mathrm{D} /$ Poss responsable de la réalisation de la racine sous la forme d'un nom. La racine insérée sous $\mathrm{V}$ monte en $v$ ' avant de monter dans la projection fonctionnelle nominalisante (deux instances du mouvement standard de la tête) :

$(51)$

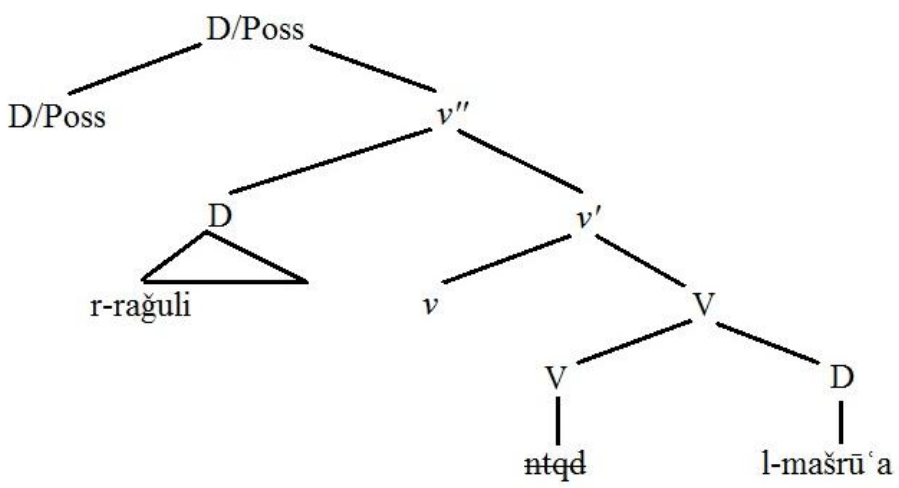

17 Ce qui est en conformité avec la généralisation de BURZIO (1986 : 178) concernant l'attribution du cas accusatif par un verbe à son argument direct interne :

Only and only the verbs that can assign the theta-role to the subject can assign (Accusative) case to an object. 
Selon cette approche, le cas accusatif attribué à l'argument interne est dû à la présence de la projection $v \mathrm{P}$. Inversement, dans le cas des mașdars accompagnés d'un complément interne introduit par une préposition, l'absence de la projection $v \mathrm{P}$ explique l'absence de cas accusatif :

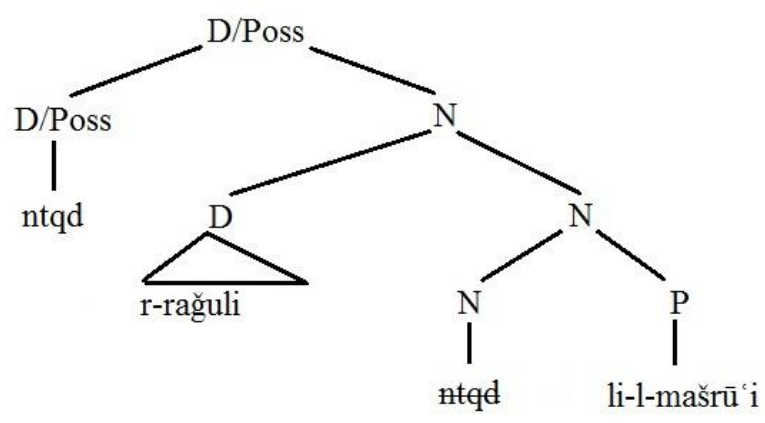

La structure ci-dessus montre que le passage de $\mathrm{V}$ à $\mathrm{N}$ prend place dans le lexique et l'absence de projection verbale explique les propriétés strictement nominales de ce type de $\operatorname{masdar}^{18}$.

Abstraction faite des différences théoriques, les deux approches expliquent les différences syntaxiques des deux mașdars par la présence ou l'absence d'une projection verbale. Reste alors à déterminer quel type de projection verbale les mașdars issus d'une nominalisation inachevée possèdent :

$$
\begin{aligned}
& \begin{array}{llll}
\text { ta'dīlu } & d \text {-dustūri } & \text { (min } & \text { țarafi } \\
\text { modification.Nom. } & \text { la-constitution.Gén. } & \text { de } & \text { part }
\end{array} \\
& \text { l-ḥukümati) askata ššsāría } \\
& \text { le-gouvernement.Gén. a calmé la-rue.Acc. }
\end{aligned}
$$

Nous suivons le principe de l'analyse proposée par ALEXIADOU (2001) (sans en adopter le cadre théorique ${ }^{19}$ ) aux déverbaux a sens processif en grec formés sur la base d'un verbe transitif direct. Les maṣdars sous (53) présentent les mêmes propriétés à savoir (i) la présence d'un argument (interne), (ii) la modification aspectuelle et de manière par un adverbe (iii) et des propriétés verbales passives. Cependant, les propriétés passives de ce type de mașdar ne signifient pas qu'ils sont formés selon un processus qui implique une passivation (du VP) même si au final, le résultat dans les deux cas est la suppression (du

18 Contrairement à KREMERS (2003), nous pensons que les mașdars issus d'une nominalisation achevée n'expriment pas des événements complexes mais des événements simples selon la terminologie de GRIMSHAW (1990)

19 Dans le cadre adopté par ALEXIADOU (2001) et KREMERS également (2003), les unités lexicales sont réduites à de simples racines; le sens est porté par les projections lexicales; et le contexte dans lequel les racines sont insérées déterminera leur nature lexicale. 
rôle) de l'argument externe (JAEGGLI 1986 ; BAKER, JOHNSON et ROBERTS 1989). En effet, si les mașdars de ce type étaient formés selon un mécanisme impliquant une passivation dont le résultat est la suppression de l'argument externe, on s'attendrait à ce qu'un verbe n'ayant pas d'argument externe ne puisse pas donner lieu à un mașdar verbal passif. Pourtant, il est possible de former le même type de mașdar en AS à partir de verbes qui n'en possèdent pas.

Depuis les travaux de PerLmutTer (1978) et BURzio (1986), on distingue parmi les verbes intransitifs deux sous classes, les inergatifs dont le sujet est un argument externe, et les inaccusatifs dont le sujet (dérivé) est un argument interne. AL-KHAWALDA (2011) étend cette distinction aux verbes arabes (en emploi) intransitifs en invoquant un ensemble de tests sémantico-syntaxiques discriminants. Nous ne citons, pour illustrer la classe des verbes inaccusatifs que celle des verbes qui entrent dans une alternance causative/non causatives sans variation morphologique, lesquels feront l'objet d'une analyse dans la dernière partie de cet article.

Les verbes concernés par cette alternance présentent un premier indice en faveur de l'existence de verbes inaccusatifs en AS. En effet, les verbes sous (54), en plus d'un emploi transitif dans lequel ils sélectionnent un argument externe (agent) et un deuxième interne (thème), entrent dans une construction intransitive sans changement morphologique, et prennent comme sujet l'argument interne ${ }^{20}(55)$ :
(54) a. tahā t-tabbāhu l-lahma
a cuit la-cuisinier.Nom. la-viande.Acc.
« Le cuisinier a cuit la viande »
b. yațbuhu ț-ṭabbāḩu d-dağāğa
cuisine le-cuisinier.Nom. le-poulet.Acc.

\begin{tabular}{|c|c|c|}
\hline ma'a & l-bașali & muddata \\
\hline
\end{tabular}
«Le cuisinier cuisine le poulet avec des oignions pendant une heure »
c. $\Sigma \bar{a} \bar{a}^{c} \quad l$-waladu l-habara
a répandu le-garçon.Nom. la-nouvelle.Acc.
«L'enfant a répandu la nouvelle »
(55) a. tahā l-lahmu
a cuit la-viande.Nom.
« La viande a cuit »

20 L'argument interne est titulaire du même rôle thématique que dans la construction transitive. Si l'on se réfère au principe UTAH (Uniform Theta-role Assignment Hypothesis), on conclut qu'il est généré dans une position interne au $\mathrm{VP}$, même dans l'emploi intransitif, là où il reçoit le rôle thème, en conformité avec le prinicipe mentionné ci-dessous :

a theta-role is assigned in the same structural position in all structures in which it is present. 


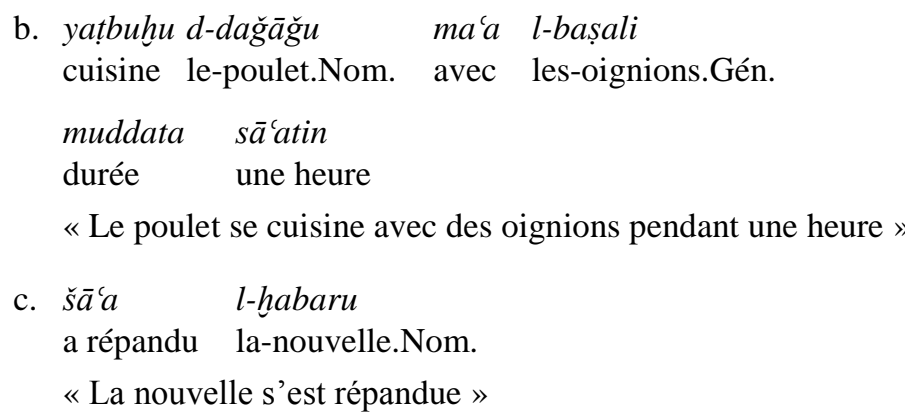

Les verbes en question dans leur emploi intransitif contrastent avec les suivants, intransitifs, dont le sujet (agent) est un argument externe, et non un argument interne ayant monté en position sujet suite à l'effacement de l'argument externe. Ces verbes n'entrent pas dans une alternance causative/non causative sans variation morphologique, et le rôle 'agent' est l'indice que le sujet est un argument externe étant donné que ce rôle sémantique est toujours attribué dans une position argumentale externe au VP :

$$
\begin{aligned}
& \text { (56) a. intafaza } \check{s} \text {-ša } a b u \\
& \text { s'est soulevé le-peuple.Nom. } \\
& \text { «Le peuple s'est soulevé » } \\
& \text { b. raqașati l-fatātu } \\
& \text { a dansé la-fille.Nom. } \\
& \text { «La fille a dansé » }
\end{aligned}
$$

Le deuxième argument donné par AL-KHAWALDA (2011 : 183) qui rapproche le sujet des verbes en (55) des arguments internes, est la possibilité pour les verbes en question de former un participé passé (ism almaf'ūl). Ce dernier a la particularité en AS de pouvoir être prédiqué des arguments internes, mais pas des arguments externes. Il peut être prédiqué (i) du cod d'un verbe transitif (57b) (ii) des sujet de verbes intransitifs inaccusatifs lesquels sont des objets internes (58), mais pas du sujet (57c) du verbe sous (57a) lequel est un argument externe :

(57) a. tarğama l-waladu l-kitāba

a traduit le garçon.Nom. le-livre.Acc.

«Le garçon a traduit le livre »

b. l-kitābu l-mutarğamu šayyiqun le-livre.Nom. le-traduit.Nom. captivant.Nom.

«Le livre traduit est captivant »

c. *l-waladu l-mutarğamu

le-garçon le-traduit.Nom. 
(58) a. akalnā l-laḥma l-mațhuwwa

nous avons mangé la-viande.Acc. le-cuit.Acc.

«Nous avons mangé la viande cuite »

b. d-dağağu l-mațbūhu ma'a l-bașali šahiyyun le-poulet.Nom. le-cuisiné.Nom. avec les-oignions.Gén. délicieux.Nom.

«Le poulet cuisiné avec des oignions est délicieux »

\section{c. kad diabnā l-habara l-muša $\bar{a}^{c} a$}

nous avons démenti la-nouvelle.Acc. le-répandu.Acc.

«Nous avons démenti la nouvelle répandue »

Les verbes sous (56) ne permettent pas la formation de participes passés de même type qui pourraient être prédiqués de leurs sujets :
(59) a. $\begin{array}{r}* \check{s}-\check{s} a b u \\ \text { le-peuple.N }\end{array}$
l-muntafizu
le-peuple.Nom. le-soulevé.Nom.
b. *l-fatātu l-marqūṣatu
la-fille.Nom. la-dansée.Nom.

Les verbes en question sont ineragtifs et permettent, contrairement aux inaccusatifs, la formation de noms d'agent (ism l-fāil) qui peuvent être prédiqués directement des sujets, lesquels sont des agents (i.e. arguments externes) :

(60) a. qatalati $\check{s}$-šurțatu (r-rağula) l-muntafiza

a tué la-police.Nom. (l-homme.Acc.) le-soulevé.Acc.

«La police a tué le (homme) révolté »
b. našarnā șuwara (l-fatāti) r-rāqisati
nous avons publié photos.Acc. (la fille.Gén.) la-danseuse.Gén.
« Nous avons publié les photos de la (fille) danseuse »

La différence entre deux types de verbes se manifeste également au niveau des maṣdars qu'ils permettent de former. En effet, les mașdars morphologiquement apparentés aux verbes intransitifs inergatifs ont toutes les propriétés des noms issus d'une nominalisation achevée :

- la modification aspectuelle et de manière s'effectue par un adjectif et non un adverbe :
(61) a. nuhayȳ ntifātatata šs $\check{s} a b i$ nous saluons soulèvement.Acc. le-peuple.Gén.
*bi- šăgā'atin /
$\check{s}$-šuğă $\bar{c}^{c} a$
didda t-tāāgiyati
avec-courage.Indéf.Gén.
courageuse.Nom./
contre le-tyran.Gén.
« Nous saluons le soulèvement courageux du peuple contre le tyran »




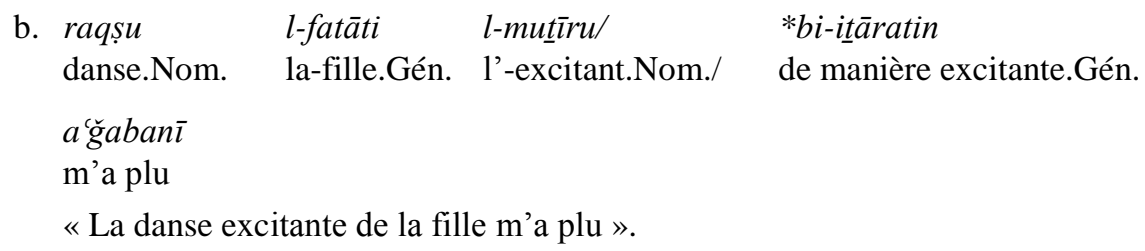

- l'agent est un actant et non un argument puisqu'il peut prendre la forme d'un adjectif de groupe :
(62) a. nuhayȳ
l-intafāzata
$\check{S}$-ša biyyata
$\check{s}$-šuğ $\bar{a} a t a$
nous saluons le-soulèvement.Acc. le-populaire.Acc.
courageuse.Nom.

didda țțāgiyati

contre le-tyran.Gén.

« Nous saluons le soulèvement populaire courageux contre le tyran »
b. r-raqșu r-ruğūliyyu ġayru muțīin
le-danse.Nom. le-masculine.Nom. pas excitant.Gén.
«La danse masculine n'est pas excitante »

- enfin, le mașdar peut être déterminé et pluralisé :

(63) a. nuhayȳi kulla l-intifāẹāti

nous saluons tous les-soulèvements.Gén.

« Nous saluons tous les soulèvement »
b. tušāhidūna l-yawma ahlā r-raqașāti
vous verrez le-jour plus bas les-danses
«Vous verrez aujourd'hui les plus belles danses »

A la différence près que les mașdars correspondants aux verbes inaccusatifs ne sont pas polysémiques étant donné qu'ils n'ont pas d'argument 'agent' et donc ne peuvent exprimer une action, ils partagent toutes les propriétés (verbales) des mașdars issus de nominalisation inachevée relevées dans la partie (2.2.1.) :

- l'aspect est exprimé par un adverbe (comme pour les verbes correspondants) et non par un adjectif :

(64) a. $\check{s} u \bar{u}^{c} u \quad$ l-habari bi-sur'atin

propagation.Nom. la-nouvelle.Gén. avec-rapidité.Gén.Indéf.

$/{ }^{*} s$-sarīi $i \quad$ yuqliqunī

/le-rapide.Gén. m'inquiète

«La propagation rapide(ment) de la nouvelle m'inquiète » 
b. al-'ulamā'u yurāqibūna marhalata tahyi

les-scientifiques.Nom. observent phase cuisson.Gén.

l-laḥmi bisur'atin / $*_{s-\text { sari }^{c}} \quad f i$

la-viande.Gén. avec-rapidité.Gén.Indéf. / le-rapide.Gén. dans

furnin munhafidatun harāratu-hū

four.Gén.Indéf. basse.Nom.Fém.Indéf. température.Nom.-Pron

«Les scientifiques observent la phase de cuisson rapide(ment) de la viande dans un four à basse température »

-les mașdars peuvent se combiner avec des prédicats qui s'accommodent de noms d'événements :

\begin{tabular}{|c|c|c|c|c|c|}
\hline (65) a. & $\begin{array}{l}\text { min bayni } \\
\text { Prép. parmi }\end{array}$ & $\begin{array}{l}\text { l-ahdātíl } \\
\text { les-événements }\end{array}$ & $\begin{array}{l}\text { l-llatī } \\
\text { qui }\end{array}$ & $\begin{array}{l}\text { fāğa'atn̄̄ } \\
\text { m'ont étonné }\end{array}$ & $\begin{array}{l}\text { s-sanata } \\
\text { l'-année }\end{array}$ \\
\hline & $\begin{array}{l}\text { l-māḍiyata } \\
\text { la-denière }\end{array}$ & $\begin{array}{l}\check{s} u y \bar{u}^{\prime} u \\
\text { propagation.Nom }\end{array}$ & & le.Gén. & ce.PronPoss \\
\hline
\end{tabular}

«Parmi les événements qui m'ont étonné l'année dernière la propagation de la nouvelle de mon divorce »

b. țahyu l-laḥmi bisur'atin

cuisson.Gén. la-viande.Gén. avec rapidité.Gén.Indéf.
fi furnin
munhafidatun
harāratu-hū
dans four.Gén.Indéf. basse.Nom.Fém.Indéf.
température.Nom.-Pron

\author{
hadatun farīdun \\ événement.Nom.Indéf. exceptionnel.Nom.Indéf.
}
«La cuisson rapide(ment) de la viande dans un four à basse température est un événement exceptionnel »

Les contrastes mentionnés ci-dessus conduisent à la conclusion que les mașdars correspondants aux verbes intransitifs inergatifs sont formés à partir d'une projection nominale alors que ceux correspondant aux verbes intransitifs inaccusatifs sont formés à partir d'une projection verbale, sans argument externe, et sans passivation aucune. Le fait qu'ils partagent les propriétés syntaxiques et sémantiques des mașdars (verbaux) correspondant à des verbes transitifs directs implique que ces derniers ne sont pas formés suite à une passivation et possèdent par ailleurs le même type de projection verbale.

Nous suivons ALEXIADOU (2001: 112 $2^{21}$ ) qui propose que les déverbaux grecs à sens processif liés à des verbes transitifs directs sont formés à partir d'une projection verbale inaccusative. Nous prenons le parti de considérer qu'il existe deux type de projections $v \mathrm{P}$ : une projection $v \mathrm{P}$ transitive, et une projection $v \mathrm{P}$ intransitive. La première se combine avec un argument externe, la seconde n'en n'a pas :

21 Cf. VAn DE Velde (2006a, 2012 manuscrit) pour le français. 
$\mathrm{v}$ transitive [+argument externe], $\mathrm{v} 1=$ Cause

$\mathrm{v}$ intransitive [-argument externe] $\mathrm{v} 2$ : devenir, se produire

Les mașdars isssus d'une nominalisation inachevée, qu'ils correspondent à des verbes transitifs directs ou intransitifs inaccusatifs, sont formés (sans l'affixe nominalisateur proposé par FASSI FEHRI 1993) à partir d'une structure qui seraient analogue à celle sous :

(66)

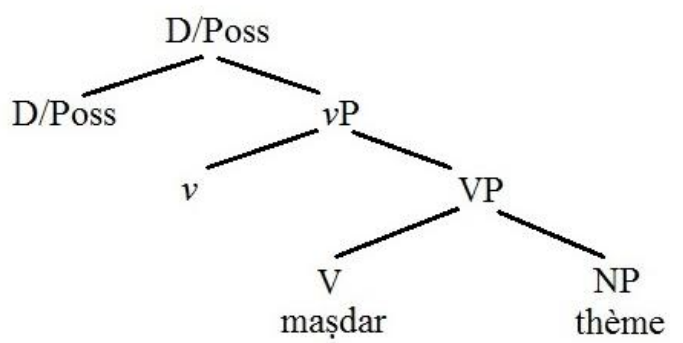

Etant donné que nous considérons que les unités lexicales (et non les projections elles mêmes) sont porteuse de sens, la structure que nous proposons sous (66) ne réduit pas les mașdars à de simples racines et insiste surtout sur l'idée que les mașdars issus de nominalisation inachevée possèdent une projection verbale inaccusative. L'absence de l'argument externe explique l'impossibilité pour l'argument thème d'avoir le cas accusatif. ${ }^{22}$ La présence d'une projection verbale inaccusative sans argument externe explique les propriétés verbales communes aux deux types de mașdars formés. Elle soulève par ailleurs la question de leur parenté sémantique.

\section{L'existence de deux types d'événements}

La définition que nous adoptons du concept «événement» est celle qui lie ce concept à celui du changement, i.e. une rupture dans un état préalablement stable. Reste à déterminer la nature du changement.

Les maṣdars liés aux verbes inaccusatifs, lesquels ont un sens événementiel, permettent d'identifier le type d'événement exprimé. Il s'agit d'évènements construits sur la base d'un changement affectant le référent de l'argument interne. Abstraction faite du la nature concrète ou abstraite du référent des arguments internes des maṣdars illustrés sous (65) et reproduits ci dessous :

$\begin{array}{lllll}\text { (67) a. min bayni } & \text { l-aḥdāti } & \text { l-latī } & \text { fāğa'atn̄i } & \text { s-sanata } \\ \text { prép. parmi } & \text { les-événements } & \text { qui } & \text { m’ont étonné } & \text { l'-année }\end{array}$

22 Il faut postuler deux mouvements qui interviennent pour donner l'ordre de surface (mașdar+argument interne). Le mașdar monte et s'incorpore à la tête du DP ; l'argument thème monte dans le Spec d'une projection fonctionnelle intermédiaire entre $v \mathrm{P}$ et $\mathrm{D} / \mathrm{Poss}$ dans laquelle il peut recevoir le cas génitif. 


$\begin{array}{llll}\text { l-mādiyata } & \check{s} u y \bar{u} u & \text { habari } & \text { țalāq } \bar{\imath} \\ \text { la-denière } & \text { propagation.Nom. } & \text { nouvelle.Gén. } & \text { divorce.PronPoss. }\end{array}$

«Parmi les événements qui m'ont étonné l'année dernière la propagation de la nouvelle de mon divorce »

b. tahyu l-lahmi bisur'atin fi

cuisson.Gén. la-viande.Gén. avec rapidité.Gén.Indéf. dans

$\begin{array}{lll}\text { furnin } & \text { munhafiḍatun } & \text { harāäratu-hū } \\ \text { four.Gén.Indéf. } & \text { basse.Nom.Fém.Indéf. } & \text { température.Nom.-Pron. }\end{array}$

hadatun farīdun

événement.Nom. exceptionnel.Nom.

« La cuisson rapide(ment) de la viande dans un four à basse température est un événement exceptionnel»

Les syntagmes formés du mașdar et l'argument interne (thème) dénotent un événement basé sur la modification de l'état ou de la localisation du référent de l'argument. Cette modification qui marque une rupture avec une situation antérieure constitue l'événement exprimé. En (67a), la modification concerne une entité abstraite qui passe d'un état (nouvelle non répandue) à un autre (nouvelle répandue), et en (67b), la modification survient dans une entité concrète dont les propriétés intrinsèques changent marquant ainsi le passage (et donc la rupture avec un situation antérieure) de l'état (viande non cuite) à celui de (viande cuite). La description sémantique qui vient d'être proposée pour l'événementialité des maṣdars liés à des verbes inaccusatifs n'a rien d'étonnant sachant que les verbes inaccusatifs eux mêmes décrivent un changement d'état qui traduit une modification survenant dans une substance représentée en syntaxe par l'argument interne. Le même sens et rapport argumental s'observent dans le cas des mașdars qui leur correspondent. Pour ce type d'évènement, nous adoptons la terminologie récente de VAN DE VELDE (manuscrit 2012) qui parle d'événements liés, lesquels expriment ce qui arrive-à l'argument interne. Les nominalisations inachevées prennent en charge l'expression de ce type d'événement. Le deuxième type identifié par VAN DE VELDE, qu'elle appelle des événements non-liés, est exprimé par les nominalisations achevées. Un événement non lié est défini comme dénotant un changement quelconque mais qui ne serait pas un changement d'état se produisant dans le référent de l'argument interne, lequel n'a plus de rapport prédicatif avec le nom formé. Un événement non lié est un événement qui décrit simplement ce qui arrive, marquant un changement (et donc une rupture avec un état antérieur) quelconque.

Dans ce qui suit, nous tentons de déterminer le type d'événement exprimé par les mașdars issus de nominalisation achevée et inachevée, liés à des verbes transitifs directs, avec en filigrane la question suivante : est-il possible, en partant des verbes eux mêmes cette fois-ci, d'identifier une base sémantique qui permet de prédire le type de mașdar qui sera formé, et par conséquent le type d'événement qui sera exprimé ?

Nous défendons l'idée qu'aux verbes suivants correspondent respectivement deux structures lexico-conceptuelles différentes qui déterminent les contraintes sur les 
nominalisations, le type d'événement exprimé de même que les autres possibilités dérivationnelles :
(68) a. qașafa l-'aduwwu l-madìnata
a bombardé l'-ennemi.Nom. la-ville.Acc.
«L'ennemi a bombardé la ville»
b. AGIR MANIERE SUR $(\mathrm{x}, \mathrm{y})$
(69) a. harrara l-ğayšu l-madīnata
a libéré l'-armée.Nom. la-ville.Acc.
«L'armée a libéré la ville »
b. tahā t-țabbāhhu l-lahma
a cuit le-cuisinier.Nom. la-viande.Acc.
« Le cuisinier cuit la viande »
c. AGIR SUR (x,y) DE SORTE QUE (DEVENIR (ETAT (y))

A la différence du français, les trois verbes permettent de former des mașdars issus des deux procédés de nominalisation, ce qui peut soulever une objection quant à la décision de leur proposer des structures lexico-conceptuelles différentes. La différence se manifeste au niveau des propriétés des participes passés qu'ils permettent de former de même que la possibilité des uns et des autres à entrer ou non dans une alternance causatives non causatives sans changement morphologique.

\subsection{La structure lexico-conceptuelle $A G I R_{\text {MANIERE }} \operatorname{SUR}(x, y)$}

La structure sous (68b) comporte le prédicat primitif transitif AGIR SUR. Ce prédicat (de même que la manière de l'action) est lexicalisé par un verbe tel que qasafa (bombader). La présence de primitif sémantique explique d'emblée de nombreuses propriétés concernant le verbe en question, à savoir la sélection d'un agent (x) et d'un complément argumental (y) (quand le prédicat est lexicalisé sous une forme verbale) lequel a un double rôle thématique. Il est à la fois patient et lieu. La rôle patient ressort de la possibilité pour le syntagme madina (ville) d'être relié au mașdar qasf (bombardement) par le verbe support hada'a (subir) qui sélectionne comme sujet des patients (70a). Il est également lieu puisqu'il peut être introduit comme complément prépositionnel du même mașdar, dépendant d'une préposition locative (70b) :
(70) a. hada'ati
l-madīnatu li-qasfin
hamağiyyin
a subi la-ville.Nom. à-bombardement.Indéf.Gén.
barbare.Indéf.Gén.
«La ville a subi un bombardement barbare »
b. al-qașfu l-amrīkiyyu 'alā l-madīnati
le-bombardement.Nom. l'-américain.Nom. sur la-ville.Gén.


$\begin{array}{ll}\text { hallafa damāran } & \text { kabīran } \\ \text { a cause dégat.Acc. } & \text { grand.Acc. }\end{array}$

«Le bombardement américain sur la ville a causé de grands dégâts »

Les deux rôles portés par le complément du verbe qașafa trouvent une explication dans le type de prédicat primitif lexicalisé AGIR SUR : en tant que prédicat d'action à deux arguments, il exprime une action transitive au sens aristotélicien, et relie un agent et un patient. Mais en tant qu'il exprime une action qui s'applique à un patient, ce type d'action requière un point d'application, ce qui indiqué par la préposition SUR, laquelle fait partie intégrante du prédicat primitif et introduit un argument auquel sera attribué le rôle sémantique de lieu. Les verbes ${ }^{23}$ qui lexicalisent la structure proposée en $(68 \mathrm{~b})$ ont une structure simple et leur analyse se fait par le biais d'une proposition simple dans laquelle l'action n'entraine pas d'état résultant conçu comme un changement de l'état du référent de l'argument $y$. L'absence d'une couche résultative, présente dans le cas des verbes illustrés sous (69), explique l'impossibilité de mobiliser le participe passé formé à partir du verbe qașafa dans un contexte qui décrit le changement d'état résultant suite à l'accomplissement de l'action :

$$
\begin{array}{llll}
\text { *baqiyati } & \text { l-madīnatu maqșūfatan } & \text { sanatan } & \text { kāmilatan } \\
\text { est restée } & \text { la-ville.Nom. bombardée.Acc. } & \text { année.Indé } & \text { entière.Indéf. }
\end{array}
$$

Le participe passé n'a pas d'emploi descriptif qualifiant mais peut par ailleurs être employé dans un contexte qui signifie un procès en cours :

$$
\begin{array}{lll}
\text { natawāğadu } & \text { qurba l-madīnati } & \text { l-maqșūfati } \\
\text { nous nous trouvonsprès } & \text { la-ville.Gén. } & \text { la-bombardée.Gén. }
\end{array}
$$

« Nous nous trouvons près de la ville bombardée »

Le changement qui se produit dans le cas d'un bombardement, et de manière générale dans le cas des verbes de contact n'est guère conçu comme un changement d'état affectant la nature même du référent du patient. Mais il y a bien un changement en termes d'entrée en contact du patient avec l'agent instigateur de l'action. Il s'agit d un changement dans la relation entre les deux référents des arguments du verbe sans changement du référent de l'argument interne. Dit autrement, et en accord avec l'analyse proposée par VAN DE VELDE pour les noms dérivés des verbes français (2012 manuscrit), l'événement exprimé par les mașdars de contact en AS est basé sur un changement qui se produit (arrive) dans l'état du monde, le cas échéant dans le rapport entre les deux entités $x$ et $y$, et non sur un changement qui se produit dans (arrive-à) l'entité $y$. Ils décrivent des événements purs, non liés, qui peuvent indifférent, et sans contrainte aucune, être présentés aussi bien du point de vue de l'agent (x) que du point de vue du patient-lieu (y). Les deux ont le même poids par rapport à l'événement exprimé, pace que les deux sont aussi, si l'on se réfère à la proposition

23 Il s'agit aussi bien de verbes transitifs directs appartenant à la classe du type hitting de FILLEMORE (1970) ((șadama-heurter), (dā'aba-caresser)) que de verbes transitifs indirects (hağama 'alä (attaquer)). Ces verbes lexicalisent uniquement la manière de l'action et présentent les mêmes propriétés relevées pour le verbe qașafa. 
lexicalisée par ces verbes, les arguments du même prédicat lexicalisé AGIR SUR. En effet, le mașdar qasf peut présenter l'événement tout aussi bien du point de vue de l'agent en l'absence du patient (73a); ou de celui du patient en l'absence de l'agent (73b) sans oublier qu'il peut tout aussi bien se délier des deux (73c) :
(73) a.
qașfu l-'aduwwi hallafa damāran
bombardement.Nom. l'ennemi.Gén. a causé dégat.Acc.Indéf.

\section{kabīran \\ grand.Acc.Indéf.}
«Le bombardement de l'ennemi a causé de grands dégâts »
b. sittata qatlā ğarrā'a l-qaşfi 'alā l-madīnati six morts suite le-bombardement.Gén. sur la-ville.Gén.
«Six morts suite au bombardement sur la ville »
c. sittata qatlā ğarrā'a l-qașfi six morts suite le-bombardement.Gén.
«Six morts suite au bombardement »

Enfin, l'absence de couche résultative dans la proposition lexico-conceptuelle lexicalisée par les verbes de contact -du moins ceux qui sont transitifs directs- explique qu'ils ne peuvent entrer dans une alternance causative/non causative neutre. Que l'on adopte l'approche ${ }^{24}$ qui stipule que la construction transitive causative est dérivée de la construction intransitive via un processus de causation illustré sous (74), ou qu'elle subit un mécanisme inverse de décausation qui produit une construction intransitive $(75)^{25}$ :

(74) Causativization rule (cf. Hale and Keyser (1986), cité également dans Shafer 2009: 661):

a. basic Lexical Conceptual Structure (LCS) of break: [become BROKEN (x)] $\rightarrow$

b. derived LCS of break: [(y) cause [become BROKEN (x)]]

(75) Detransitivization rule (Grimashaw 1982)
a. causative: [(x) cause [become $\operatorname{BROKEN}(\mathrm{y})]] \rightarrow$
b. anticausative: [become $\operatorname{BROKEN}(\mathrm{y})]$

24 Voir LAKoff $(1968,1970)$; Williams (1981) ; HALE \& Keyser $(1986,1987)$; HARLEy (1995) ; PESETSKY (1995) entre autres.

25 C'est l'approche que nous prônons, préconisée par GRIMSHAW (1982); CHIERCHIA (1989/2004); LEVIN \& RAPPAPORT $(1994,1995)$ et REINHART (2002) entre autres. Les arguments qui motivent ce choix sont développés dans la partie 4.2. 
une couche résultative est nécessaire afin de permettre l'alternance, ce que les verbes décrits dans cette partie ne possèdent pas. A défaut, la suppression de l'agent ne peut avoir lieu, et la formation d'une construction intransitive neutre qui décrirait le changement d'état survenant dans l'entité $y$ en lexicalisant la couche résultative (DEVENIR (ETAT (y)) est bloquée. Les différentes possibilités (et impossibilités) dérivationnelles mentionnées pour les verbes de ce type, qu'ils s'agisse (i) du sens du participe passé, (ii) du type (et du sens) du mașdar formé et (iii) de l'alternance causative/non causative neutre montrent qu'ils lexicalisent une proposition simple et expriment un événement non lié basé sur un changement se produisant dans le monde mais non affectant la nature du référent de l'argument interne $\mathrm{y}$.

Cependant, le verbe qașafa, comme tous les verbes (transitifs directs) de contact en AS donnent lieu aussi à la formation d'un mașdar issu d'une nominalisation inachevée et entretiennent par conséquent un rapport prédicatif avec leur uniquement argument, l'argument interne. Cette possibilité les rapproche des verbes qui seront décris dans la partie suivante, lesquels lexicalisent une structure lexico-conceptuelle complexe. Ces derniers, à leur tour, permettent la formation de mașdars ayant des propriétés nominales, mais avec une contrainte, que les précédents ne présentent pas, et qui appuie l'hypothèse que les verbes suivants, même dans leur version transitive, lexicalisent la couche résultative, i.e. le changement survenu dans l'entité y lequel est à la base de l'événement exprimé par les mașdars qu'ils forment.

\subsection{La structure AGIR SUR (x,y) DE SORTE QUE (DEVENIR (ETAT (y))}

Comparée à la précédente, la structure lexico-conceptuelle lexicalisée par les verbes suivants, reproduite sous (76d), est complexe et lie par un primitif causal (DE SORTE QUE) deux propositions, chacune mobilise un prédicat différent :

$$
\begin{aligned}
& \text { (76) a. 'addala l-maliku d-dustüra } \\
& \text { a modifié le-Roi.Nom. la-constitution.Acc. } \\
& \text { «La Roi a modifié la constitution » } \\
& \text { b. harrara l-amrīkiyyūna l-madīnata } \\
& \text { ont libéré les-américains la-ville.Acc. } \\
& \text { «Les américains ont libéré la ville » } \\
& \text { c. tahā t-tabbāhnu l-lahma } \\
& \text { a cuit la-cuisinier.Nom. la-viande.Acc. } \\
& \text { « Le cuisinier a cuit la viande » }
\end{aligned}
$$

\section{d. AGIR SUR (x,y) DE SORTE QUE (DEVENIR (ETAT (y))}

La première proposition relie par le primitif AGIR deux arguments $(\mathrm{x}, \mathrm{y})$ et exprime une action transitive qui passe de l'agent (x) sur la patient (y). Cependant, le propre de ce type d'action est d'affecter l'entité y laquelle figure dans la deuxième proposition enchâssée comme argument du primitif DEVENIR. La deuxième proposition exprime le 
changement d'état résultant qui se produit dans l'entité $y$, interprétée comme thème. L'entité $y$ qui représente l'argument interne des verbes qui lexicalisent cette structure (ou du moins une partie) y figure deux fois comme argument de deux primitifs différents, ce qui explique le double rôle qu'il reçoit. La différence majeure entre les verbes reliés à cette structure est que même dans leur version verbale, ils lexicalisent la couche résultative, c'est-à-dire le changement survenu dans l'entité $y$.

Le premier argument en faveur de l'idée que les verbes sous (76) lexicalisent le résultat de l'action menée par l'agent et donc le changement d'état du référent de leur argument interne, est qu'ils permettent sans exception aucune de former des participes passés à sens résultatif (ism almaf ${ }^{\prime} \bar{u} l$ ), contrairement aux verbes étudiés dans la partie précédente :

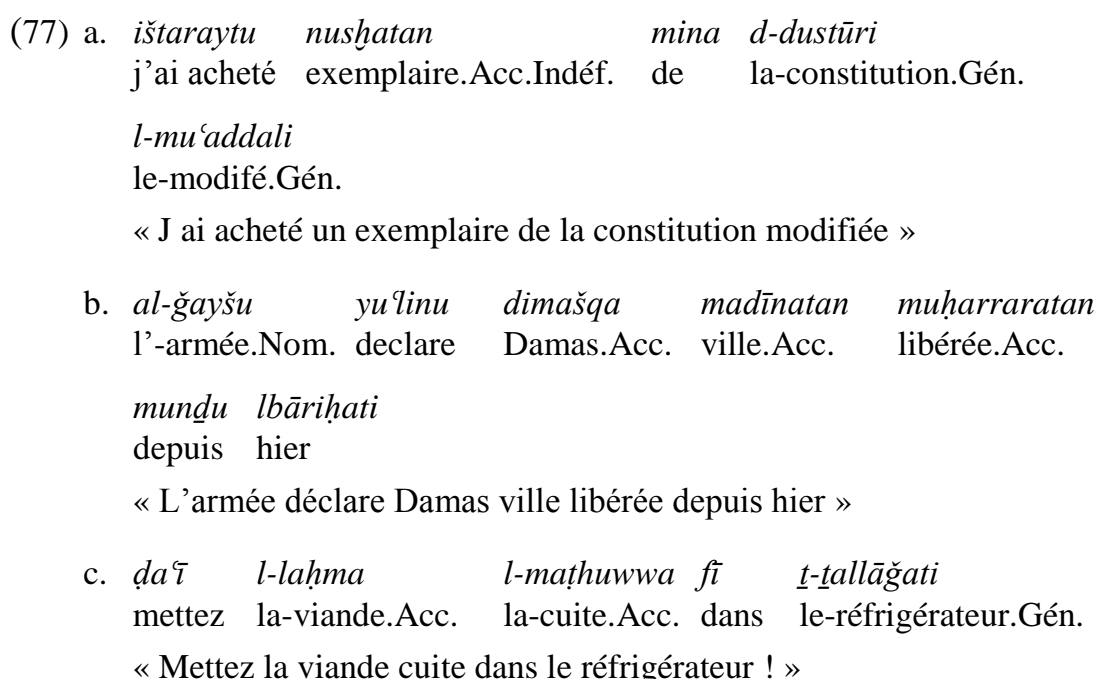

Le deuxième argument qui montre le caractère périphérique de la couche contenant l'agent (et son action sur le patient) -comparée à la couche résultative- même dans la lexicalisation verbale, est la possibilité pour certains verbes d'entrer dans une alternance causative/non causative sans variation morphologique. Les approches qui prônent l'idée de dériver la construction intransitive qui présente le changement sans source agentive, de la construction agentive via une détransitivation, s'accordent sur la contrainte de l'affectation du référent du complément par l'action de l'agent. Cette contrainte opère également dans les constructions passives moyennes et dans la formation d'une construction génitive avec antéposition du nom. Les contrastes suivants illustrent la contrainte de l'affectation :

\section{Affectedness constraint (Anderson (1977):}

If the head noun does not express an action which affects, i.e. modifies, the state of the object, the latter cannot occur in the prenominal position.

(78) the book's destruction vs. *the book's discussion 


\section{Affectedness constraint (Jaeggli 1986):}

If a complement of $\mathrm{X}$ is unaffected, it is impossible to eliminate the external role of $\mathrm{X}$.

$$
\text { This wood slits easily } \quad \text { vs. *This cat chases easily }
$$

La possibilité de former un participe passé résultatif (ism almaf ${ }^{\prime} \bar{u} l$ ) à partir des verbes sous (77) montrent qu'ils expriment des actions qui ont pour effet de produire un changement dans la nature du thème. Ce dernier est donc affecté par les actions qui trouvent leur source dans l'agent. Si l'on se base sur la contrainte de l'affectation donnée comme principe pour éliminer l'argument externe, on s'attendrait à ce que les trois verbes sous (76) entrent dans une alternance causative/non causative sans variation morphologique. Or seul le dernier verbe țaha (cuire), et pour donner d'autres exemples gala (bouillir), et $\check{s} \bar{a} c a$ (répandre) admettent la suppression de l'agent afin de présenter le changement affectant le référent du complément sans source. La différence entre les verbes de changement d'état qui admettent la suppression de l'agent et ceux qui ne l'admettent pas ne signifient pas que les derniers lexicalisent autre chose que la couche résultative, et donc autre chose que le changement d'état de l'entité $y$.

Pour les verbes de changement d'état, la différence entre d'une part 'addala (modifier), harrara (libérer) et d'autre part țaha (cuire) et $\breve{s}^{\prime} a$ (répandre) est une différence au niveau de la structuration interne des procès exprimés. La contrainte qui semble opérer en AS au niveau des verbes à alternance causative/non causative sans variation morphologique est que seuls les procès (i) visés et (ii) non contrôlés depuis leur début à leur fin par l'agent ${ }^{26}$, admettent la suppression de ce dernier.

En effet, aussi bien 'addala (modifier) que harrara (libérer) expriment des procès visés dans le sens où le changement de l'état de l'entité $y$ est une fin à laquelle tend l'agent. Par contre, pour atteindre cette fin, l'implication de l'agent dans les différentes phases du processus est requise, c'est dans ce sens qu'il faut comprendre la deuxième contrainte. A titre d'exemple, il ne suffit pas de réunir une armée, de la positionner près d'une ville occupée et de vouloir la libérer pour qu'elle le devienne. La libération d'une ville occupée, et donc le changement de l'état de l'entité, n'ont lieu que si l'agent agit du début du processus jusqu'à la fin, à savoir l'élimination du dernier occupant. Il en va de même pour l'action 'addala (modifier) : l'implication de l'agent (i.e. le contrôle) débute avec le début de l'action et s'étend jusqu'à l'accomplissement du changement d'état. Dans les deux cas de figure, il n'existe pas de phase qui se déroule sans l'agent. Ce qui rend son effacement impossible et explique par conséquent l'impossibilité pour les verbes de ce type, alors qu'ils expriment un changement d'état et mettent l'accent sur l'information codée dans la couche résultative, de faire l'économie de l'agent.

Les propriétés aspectuelles des procès țahā (cuire) et $s^{a} \bar{a} a$ (répandre) sont différents. Le changement d'état de l'entité $y$ est une fin visée par l'agent, lequel n'est pas impliqué dans les différentes phases. Le processus de cuisson débute avec la préparation des conditions requises

26 La contrainte (ii) relevée par VAN DE VELDE (2011) pour certains verbes français, permet de rendre compte des propriétés des différents verbes concernés par cette propriété en AS qui ne sont pas nombreux car la construction est moins productive qu'en français ou en anglais. 
pour le lancement de la cuisson. L'agent réunit les conditions nécessaires pour que la cuisson se fasse, et au moins une des phases du processus, notamment celle du changement d'état, se déroule sans son intervention. La structuration interne du procès $5 \bar{s}^{c} a$ (répandre), dont l'état résultant est visé par l'agent qui en est la source, présente également une phase qui se déroule sans l'implication, et par conséquent, sans le contrôle de l'agent. Pour qu'une nouvelle devienne répandue, il suffit de la transmettre à un autre agent lequel la transmettra à son tour à d'autres agents et ainsi de suite qui la relayeront. L'implication de l'agent-source s'arrête à un moment donné sans que cela empêche l'accomplissement du changement d'état.

Le fait que l'implication de l'agent débute avec le début de l'action mais ne s'étend pas jusqu'à l'accomplissement du changement d'état permet aux verbes concernés par cette propriété aspectuelle de faire aisément l'économie de la couche agentive et de ne retenir que la couche résultative, laquelle comporte une phase qui se déroule de manière autonome indépendamment d'un agent contrôleur. L'autonomie d'au moins une des phases des processus exprimés explique la possibilité de combiner les verbes concernés par cette propriété aspectuelle avec le verbe taraka (laisser) et autres variantes qui marquent, même en la présence de l'agent, la non implication de ce dernier dans le déroulement du changement d'état (i.e. le devenir) de l'entité $y$ :

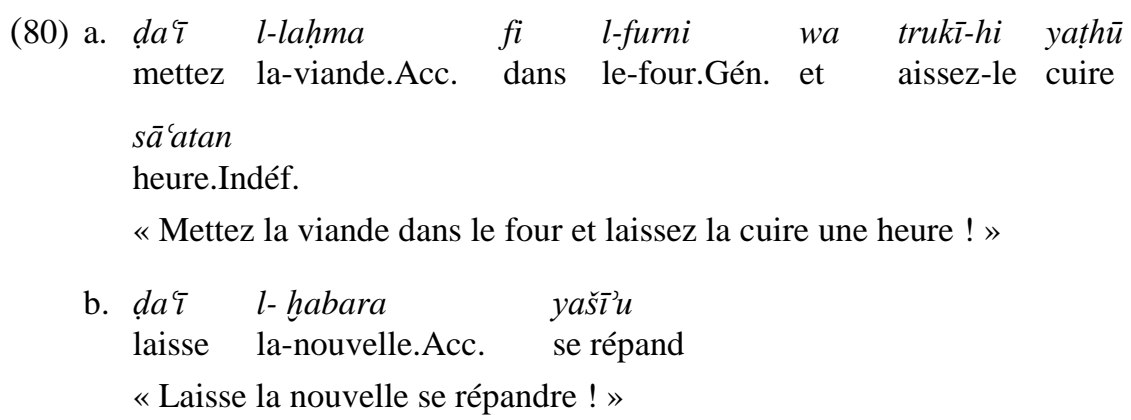

Pour revenir aux verbes du type 'addala (modifier), le fait qu'ils ne permettent pas la suppression de la couche agentive ne signifie pas qu'ils expriment des actions sans changement d'état, ou qu'ils lexicalisent la couche agentive, contrairement aux verbes de changement d'état du type tahā (cuire) qui lexicalisent le résultat sans la couche agentive. Les deux types de verbes lexicalisent, même dans leur variante transitive, uniquement le résultat. Ils ont une structure conceptuelle complexe analogue, mais aspectuellement différente, qui comporte un prédicat primitif DEVENIR, lequel prend pour unique argument le thème $(\mathrm{y})$, laissant en dehors de la prédication l'agent $(\mathrm{x})$. Le poids des deux couches est différent, le résultat, et donc le devenir de l'entité (y) semble être la prédication la plus importante. Cette prédication n'est rien d'autre qu'un événement lié basé sur un changement survenu dans une entité. Les contraintes sur les mașdars qu'ils forment confirment l'hypothèse que le poids de la couche résultative est plus important.

Nous avions remarqué que les mașdars en rapport avec des verbes qui lexicalisent une structure lexico-conceptuelle simple expriment un événement qui peut être présenté aussi bien du point de vue de l'agent (x) que du point de vue du patient-lieu (y). Les deux ont le même poids par rapport à l'événement exprimé, pace que les deux sont aussi, si l'on se 
réfère à la proposition lexicalisée par ces verbes, les arguments du même prédicat lexicalisé AGIR SUR.

Une contrainte intéressante se manifeste au niveau des mașdars en lien avec des verbes qui lexicalisent une structure lexico-conceptuelle complexe. Comme ils ont une structure complexe qui associe le prédicat AGIR SUR à un agent, mais que cet agent est périphérique, effaçable dans le cas verbes dont l'agent ne contrôle pas toutes les phases du processus, il est possible de former un mașdar issu d'une nominalisation achevée dont la particularité est l'absence de structure argumentale. L'agent y figure mais simplement comme complément sémantique et non comme argument, pouvant même être instancié sous la forme d'un adjectif de groupe. Nous obtenons donc la configuration illustrée sous :

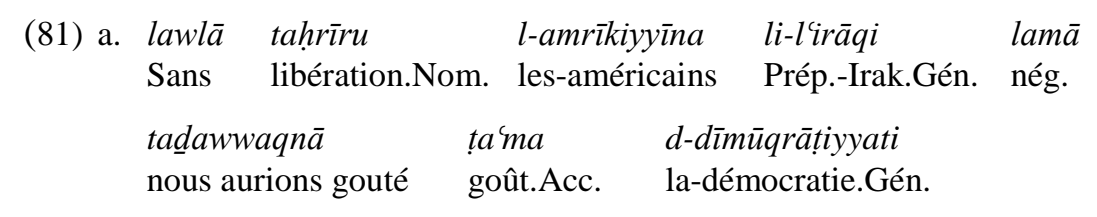

«Sans la libération des américains de l'Irak nous n'aurions pas gouté à la démocratie »
a'. $\bar{u} l \bar{a}$
hutụwāti
t-tahrīri
l-amrīkiyyi
premiers pas.Gén.
libération.Gén.
l'-américain.Gén.
li-l-madīnati tatadammanu
Prép.la-ville.Gén. inclut
«Les premières étapes de la libération américaine de l'Irak incluent... »
b. u'îbu 'alay-ka išăáataka
je reproche Prép-toi mașdar de répandre-toi
li-l-habari
«Je te reproche d'avoir répandu la nouvelle »
Prép.la-nouvelle.Gén.

Dans le cas d'un mașdar issu d'une nominalisation achevée à partir d'un verbe sans couche résultative, nous avions également remarqué que la présence du complément prépositionnel qui renvoie à l'argument interne du verbe n'est pas dépendante de celle du complément agent. Le mașdar n'entretient plus de rapport prédicatif avec les constituants qui l'accompagnent si bien qu'il peut indifféremment se délier de l'un ou de l'autre.

Par contre, dans le cas des mașdars illustrés sous (81), le second complément prépositionnel ne peut être instancié qu'en la présence de l'agent alors même que ce dernier n'est pas un argument puisqu'il peut être réalisé sous la forme d'un adjectif de groupe (81a'). La réalisation de l'agent sous une forme adjectivale est le signe d'un processus de nominalisation achevée que confirme également la catégorie des prédicats de manière et d'aspect qui ne peut être que nominale. La contrainte relevée est illustrée ci-dessous :
(82) a. *lawlā t-tah́rīru
li-lírāqi
lamā tadawwaqnā
Sans libération.Nom. Prép.-Irak.Gén. nég. nous aurions gouté 


$$
\begin{aligned}
& \text { țúma d-dīmūqrātịyati } \\
& \text { gout.Acc. la-démocratie.Gén. } \\
& \text { b. *uîbu 'alay-ka l-išā'ata li-l-habari } \\
& \text { je reproche Prép.-toi masdar de répandre Prép.la-nouvelle.gén. }
\end{aligned}
$$

La double question soulevée par cette contrainte est la suivante : qu'est-ce qui bloque la réalisation de l'argument interne du verbe correspondant sous la forme d'un adjoint prépositionnel au génitif ? Et comment relier cette contrainte à la structure lexicoconceptuelle des verbes auxquels les masdars sont liés?

Si l'on admet que les verbes causatifs de changement d'état lexicalisent (même dans leur version transitive) la couche résultative (DEVENIR (ETAT (y)), le propre de cette couche est de décrire le changement survenu dans l'entité y, laquelle est l'unique argument du prédicat primitif DEVENIR. Le statut argumental de l'entité y se traduit en syntaxe par une réalisation en tant qu'argument, et non en tant qu'adjoint prépositionnel. D'un point de vue sémantique, le concept exprimé par la couche résultative est celui d'un événement lié basé sur le changement survenu dans le référent de l'entité y. Pour exprimer ce type d'événement, il faut passer par une constrction dans laquelle le nom tête entretient un lien prédicatif avec l'argument interne du verbe qui représente l'entité y. La réalisation de l'argument interne du verbe correspondant sous la forme d'un adjoint prépositionnel (en l'absence de l'agent) reste l'apanage des mașdars liés à des verbes qui expriment un changement quelconque, lequel peut être présenté aussi bien du point de vue de l'agent que du patient, parce que l'événement exprimé est un événement non lié. Pour revenir aux mașdars correspondants à des verbes causatifs de changement, les raisons, aussi bien sémantiques que syntaxiques inhérentes à la couche résultative que les verbes lexicalisent, expliquent l'agrammaticalité des exemples sous (82) qui contrastent avec les suivants dans lesquels l'entité y affectée par le changement est réalisée en tant qu'argument. Ce mode de réalisation est conforme aux exigences conceptuelle et syntaxique de la couche lexicalisée:

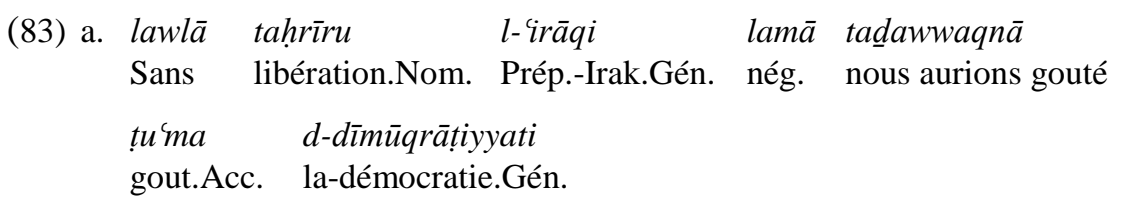

«Sans la libération de l'Irak, nous n'aurions pas gouté à la démocratie »
b. u'̌bu 'alay-ka išăćata
je reproche Prép.-toi masdar de répandre
l-habari
« Je te reproche la diffusion de la nouvelle »
Prép.la-nouvelle.gén. 


\section{Conclusion}

Pour récapituler, nous dirons que les verbes d'action transitifs directs se croisent. Contrairement au français, aussi bien qașafa (bombarder) que le couple harrara (libérer) et tahā (cuisson) peuvent donner lieu à des mașdars issus d'un processus de nominalisation achevée et inachevé. Ce qui implique de chercher les différences ailleurs. En effet, à partir des verbes de contact non causatifs de changement d'état on peut former un mașdar à propriétés verbales qui entretient un rapport prédicatif avec l'argument interne du thème, mais il peut aussi donner lieu à la formation d'un mașdar à propriétés nominales qui peut se délier aisément de cet argument et présenter l'événement exprimé, sans contrainte aucune, aussi bien du point de vue de l'agent que de celui qui le subit, le patient. C'est cette deuxième propriété qui identifie en AS les mașdars issus d'une nominalisation achevée, lesquels expriment un événement non lié et sont en rapport avec des verbes à couche lexico-conceptuelle simple. Les verbes causatifs de changement d'état peuvent donner lieu à des mașdars à propriétés nominales, mais avec une contrainte majeure qui les différencient des précédents, à savoir l'impossibilité de réaliser l'argument interne sous la forme d'un adjoint prépositionnel en l'absence de l'agent, et de s'aligner par conséquent sur les mașdars qui expriment des événements non liés. Etant donné que le référent de l'argument interne du verbe est affecté par le changement, ce dernier doit être réalisé comme argument. Cette contrainte trouve son explication en partant d'une analyse lexicoconceptuelle des verbes laquelle permet également d'expliquer les possibilités (et les impossibilités) dérivationnelles, telles que la formation d'un participe passé résultatif et l'alternance pour un verbe donné entre un emploi causatif et non causatif sans variation morphologique.

\section{Bibliographie}

Alexiadou, Artemis / Melita, Stavrou. 1998. "On Derived Nominals in Greek”. In: JosePH (et al., eds.) 1998, 2: 101-130.

-. 2001. Functional Structure in Nominals and Ergativity. Amsterdam: John Benjamins.

Alexiadou, A. / Anagnostopoulou, E. / Everaert, M. (eds.). 1989/2004. The unaccusativity puzzle: explorations of the syntax-lexicon interface. Oxford, UK: Oxford University Press.

Al-Khawalda, Mohammad. 2011. "Arabic Versus English Ergative Verbs". Damascus University Journal, 27/3+4: 163-185.

ANDERSON, Mona. 1977. "NP Pre-posing in Noun Phrases". Proceedings of NELS, 8: 12-21.

Arjoca-Ieremia, A. / Avezard-Roger, C. / Goes, J. / Moline, E. / Tinu, A. (eds.) 2011. Temps, aspect et classes de mots : études théoriques et didactiques. Arras: Artois Presses Université.

BAKER, Mark / KYle, Johnson / RoBERTs, Ian. 1989. "Passive Arguments Raised". Linguistic Inquiry, 20: 219-225.

BARDEAS, Suzanne. 2009. The Syntax of the Arabic DP. PhD thesis, University of York, United Kingdom.

Borer, H. (ed.). 1988. Proceedings of the West Coast Conference on Formal Linguistics (WCCFL), 7. Stanford: CSLI.

Bresnan, J. (ed.). 1982. The Mental Representation of Grammatical Relations. Cambridge, MA: MIT Press. 
BURZIO, Luigi. 1986. Italian Syntax. A Government and Binding Approach. Dordrecht: Reidel.

CHIERCHIA, Gennaro. 1989/2004. "A semantics for unaccusatives and its syntactic consequences". In: AlEXIADOU / ANAGNOSTOPOUlOU / EVERAERT (eds.) 1989/2004: 22-59.

DAHECH, Dhouha. 2007. "Les nominalisations déverbales en arabe et en français: Etude exploratoire des critères de classement". Les cahiers du CRISCO 23: 128-138.

—. 2008. "Les déverbaux et l'expression de l'événement en arabe et en français“, Actes du colloque Linguistique des événements, 75-98. Tunis: Université de Carthage.

FASSI FEHRI, Abdelkader. 1993. Issues in the structure of Arabic clauses and words. Dordrecht: Kluwer Academic Publishers.

FilLmORE, Charles. 1970. "The Grammar of Hitting and Breaking”. In: JaCOBS / RoSEnBAUM 1970: 120-133.

GRIMSHAw, Jane. 1990. Argument structure. Cambridge, Mass: The MIT Press.

- 1982. "On the lexical representation of Romance Reflexive Clitics". In: BRESNAN (ed.) 1982: 87148 .

HALE, Keneth / KeYSER, Samuel Jay. 1986. "Some Transitivity Alternations in English". ASJU XX/3: 605-638.

—. 1987. "A View from the Middle". Lexicon Project Working Papers 10. Cambridge, Mass.: MIT Press.

HARLEY, Heidi. 1995. Subjects, Events and Licensing. PhD thesis, MIT.

Jacobs, R. / Rosenbaum, P. (eds.). 1970. Readings in Englisch Transformational Grammar. Waltham, Mass: Ginn and company.

JAEGGLI, Osvaldo. 1986. "Passive". Linguistic Inquiry, 17: 587-622.

Joseph, B. / Horrocks, G. / PhilipPaki-Warburton, I. (eds.). 1998. Themes in Greek Linguistics. Amsterdam: Benjamins.

Kremers, Joost. 2003. The Noun Phrase in Arabic: A Minimalist Approach. PhD thesis (LOT Dissertation Series 79), University of Nijmegen.

-. 2007. "Masdar formation". In: MotZKI / DiTTERs (eds.) 2007: 475-499.

KunO, S. (ed.). 1968. Report NSF-20. Cambridge, MA: Harvard University Press.

LAKOFF, George. 1968. "Some Verbs of Change and Causation. Mathematical Linguistics and Automatic Translation". In: KuNO (ed.) 1968: 1-27.

- 1970. Irregularities in Syntax. New York: Holt, Rinehart and Winston.

Lecarme, J. / Lowenstamm, J. / Shlonsky, U. (eds.). 1996. Studies in Afro-Asiatic Grammar. The Hague: Holland Academic Graphics.

Levin, Beth / Hovav, Malka Rappaport. 1995. Unaccusativity. At the Syntax-Lexical Semantics Interface. Cambridge, Mass.: MIT Press.

-. 2005. Argument realization. Cambridge, UK: Cambridge University Press.

—. 1994. "A Preliminary Analysis of Causative Verbs in English”. Lingua, 92: 35-77.

MARKANTONATOU, Stella. 1995. "Modern Greek Deverbal Nominals, an LMT Approach". Journal of Lingusitics, 31: 267-299.

MoHAmmad, Mohammad. 1988. "On the parallelism between IP and DP”. In: Borer, H. (ed.) 1988, 7: 241-254. Stanford: CSLI

Motzki, H. / DitTers, E. (eds.). 2007. Approaches to Arabic Linguistics. Studies in Semitic Languages and Linguistics. Leiden: Brill.

OrSnes, Bjarne / Markantonatou, Stella. 2002. "Group Adjectives". Journal of Greek Linguistics, 3: 139-178.

OuHALla, Jamal. 1988. The Syntax of Head Movement: A Study of Berber. London: University College, $\mathrm{PhD}$. dissertation.

—. 1996. "The Construct State in Berber". In: LeCARME / Lowenstamm / ShLONSKy (eds.) 1996: 278-301

$J A I S \bullet 15$ (2015): 19-57 
PerlmutTer, David. 1978. "Impersonal Passives and the Unaccusative Hypothesis". Proceedings of the Fourth Annual Meeting of the Berkeley Linguistic Society, 157-189.

PESETSKY, David. 1995. Zero Syntax. Experiencers and Cascades. Cambridge, Mass.: MIT Press.

ReInHart, Tanya. 2002. "The Theta System - An Overview”. Theoretical Linguistics, 28: 229-290.

RITTER, Elizabeth. 1988. “A head-movement approach to construct-state noun phrases”. Linguistics, 26: 909-929.

—. 1991. "Two functional categories in noun phrases: Evidence from Modern Hebrew". In: RотнSTEIN (ed.) 1991: 37-62.

—. 1993. "Where's gender?" Linguistic Inquiry, 24: 795-803.

- 1995. "On the Syntactic Category of Pronouns and Agreement". Natural Language and Linguistic Theory, 13: 405-443.

Rothstein, S. (ed.). 1991. Perspectives on Phrase Structure. Special issue of Syntax and Semantics, vol. 25. San Diego: Academic Press.

SCHÄFER, Florian. 2009. "The Causative Alternation”. Language and Linguistics Compass, 3/2: 641681.

VAN DE Velde, Danièle. 2006a. Grammaire des événements. Lille: Presses Universitaires du Septentrion.

—. 2006b. "Les adjectifs de groupe". Travaux de linguistique, 53: 135-154.

— . 2011. "Le rapport des prédicats d'état résultant à l'agent du changement d'état : une analyse conceptuelle et aspectuelle”. In: ARJOCA-IEREMIA (et al., eds.) 2011: 265-276.

— . 2012. "Supplément à la grammaire des événements". Manuscrit.

WiLLIAMS, Edwin. 1981. “Argument structure and morphology”. The Linguistic Review, 1: 81-114. 